\title{
MYSM1 maintains ribosomal protein gene expression in hematopoietic stem cells to prevent hematopoietic dysfunction
}

\author{
Jad I. Belle, ${ }^{1,2}$ HanChen Wang,,$^{1,2,3}$ Amanda Fiore, ${ }^{1,2}$ Jessica C. Petrov, ${ }^{1,2}$ Yun Hsiao Lin, ${ }^{1,2}$ \\ Chu-Han Feng, ${ }^{1,2}$ Thi Tuyet Mai Nguyen, ${ }^{4}$ Jacky Tung, ${ }^{1,2}$ Philippe M. Campeau, ${ }^{4}$ Uta Behrends, ${ }^{5}$ \\ Theresa Brunet, ${ }^{6}$ Gloria Sarah Leszinski,, ${ }^{6,7}$ Philippe Gros,, ${ }^{2,8,9}$ David Langlais,, ${ }^{2,3,10}$ \\ and Anastasia Nijnik ${ }^{1,2}$ \\ Department of Physiology, ${ }^{2}$ McGill University Research Centre on Complex Traits, and ${ }^{3}$ Department of Human Genetics, \\ McGill University, Quebec, Canada. ${ }^{4}$ Centre Hospitalier Universitaire St. Justine Research Center, University of Montreal, \\ Quebec, Canada. ${ }^{5}$ Childrens' Hospital and 'Institute of Human Cenetics, Technische Universität München (TUM), \\ Munich, Germany. ${ }^{7}$ Institute of Human Genetics, Helmholtz Zentrum München, Neuherberg, Germany. ${ }^{8}$ Department of \\ Biochemistry and ${ }^{9}$ The Rosalind and Morris Goodman Cancer Research Centre, McGill University, Quebec, Canada. ${ }^{10} \mathrm{McGil}$ \\ University Genome Centre, Montreal, Quebec, Canada.
}

Ribosomopathies are congenital disorders caused by mutations in the genes encoding ribosomal and other functionally related proteins. They are characterized by anemia, other hematopoietic and developmental abnormalities, and p53 activation. Ribosome assembly requires coordinated expression of many ribosomal protein $(R P)$ genes; however, the regulation of $R P$ gene expression, especially in hematopoietic stem cells (HSCs), remains poorly understood. MYSM1 is a transcriptional regulator essential for HSC function and hematopoiesis. We established that HSC dysfunction in Mysm1 deficiency is driven by p53; however, the mechanisms of p53 activation remained unclear. Here, we describe the transcriptome of Mysm1-deficient mouse HSCs and identify MYSM1 genome-wide DNA binding sites. We establish a direct role for MYSM1 in RP gene expression and show a reduction in protein synthesis in Mysm1-1- HSCs. Loss of p53 in mice fully rescues $\mathrm{Mysm}^{-1-}$ anemia phenotype but not $R P$ gene expression, indicating that $R P$ gene dysregulation is a direct outcome of Mysm1 deficiency and an upstream mediator of $\mathrm{Mysm1}^{-1-}$ phenotypes through p53 activation. We characterize a patient with a homozygous nonsense MYSM1 gene variant, and we demonstrate reduced protein synthesis and increased p53 levels in patient hematopoietic cells. Our work provides insights into the specialized mechanisms regulating $R P$ gene expression in HSCs and establishes a common etiology of MYSM1 deficiency and ribosomopathy syndromes.

Authorship note: JIB and HCW contributed equally to this work.

Conflict of interest: The authors have declared that no conflict of interest exists.

Copyright: (c) 2020, American Society for Clinical Investigation.

Submitted: March 15, 2019

Accepted: May 21, 2020

Published: July 9, 2020.

Reference information: JCI Insight 2020;5(13):e125690.

https://doi.org/10.1172/jci.

insight.125690.

\section{Introduction}

It is widely recognized that the maintenance of hematopoietic stem cells (HSCs) and hematopoietic homeostasis is dependent on normal regulation of ribosome function and protein synthesis $(1,2)$. This requires coordinated expression of many genes, including 80 genes encoding the protein components of the ribosome (i.e., ribosomal protein genes; RP genes) and the genes encoding regulators of ribosome biogenesis and protein translation. Mutations in such genes cause congenital disorders, including Diamond-Blackfan anemia (DBA) (3), Shwachman-Diamond syndrome (SDS) (4), and other related conditions. Additionally, $5 \mathrm{q}^{-}$syndrome is linked to $R p s 14$ gene deletions $(5,6)$. Collectively, these diseases are referred to as ribosomopathies and are characterized by hematopoietic pathologies, including anemia, pancytopenia, BM failure, and increased risk of hematologic malignancies, as well as growth, skeletal, and other developmental abnormalities in some cases $(7,8)$. At the molecular lev$\mathrm{el}$, these pathologies are associated with the activation of $\mathrm{p} 53$ stress response as a result of ribosome dysfunction in affected cells (9-13). Despite the strong association of ribosomal defects with hematopoietic abnormalities, the cell-intrinsic mechanisms that regulate $R P$ gene expression specifically in HSCs remain poorly understood. 
MYSM1 is a chromatin-interacting protein with deubiquitinase (DUB) catalytic activity comprising SANT, SWIRM, and catalytic MPN domains $(14,15)$. A recently described familial MYSM1 deficiency syndrome is characterized by BM failure, with anemia, leukopenia, and complex developmental aberrations (16-18), although its molecular mechanisms remain poorly understood. We and others demonstrated the essential role of MYSM1 in maintaining HSC function and hematopoiesis in mouse models (19). Murine MYSM1 deficiency results in loss of HSC quiescence (20), increased apoptosis of multipotent progenitors (MPPs) (21), and a severe depletion of most downstream hematopoietic lineages, including erythrocytes (19), lymphocytes (22-26), and DCs (27). In both humans and mice, MYSM1 deficiency can also present with skeletal and other developmental abnormalities $(18,19,22)$. Overall, this suggests that MYSM1 functions are conserved between humans and mice, and highlights the biomedical significance of understanding MYSM1 mechanisms of action.

Recent studies indicated that MYSM1 promotes the expression of genes encoding several hematopoietic lineage-specification transcription factors, including Ebfl in B cell progenitors (22), Id2 in NK cell progenitors (24), Flt3 in DC precursors (27), and Gfi1 in the HSC and MPP cells (20). It was suggested that MYSM1 promotes the expression of these genes via deubiquitination of histone H2A (H2A-K119ub) at their promoters and via local recruitment of other transcriptional regulators such as E2A at the Ebf1 locus (22). Importantly, all previous studies focused on a small number of putative MYSM1-regulated genes. The lack of systematic and genome-wide studies of MYSM1-regulated loci remains a major limitation for the understanding of MYSM1 biological functions and mechanisms of action.

In recent work, we demonstrated that MYSM1 is an essential negative regulator of the p53 stress response pathway in hematopoietic cells $(21,28,29)$, with Mysm1 deficiency resulting in a strong induction of p53 stress response genes in hematopoietic cells $(21,28)$. Strikingly, p53 ablation in $M y s m 1^{1^{-1}} p 53^{-/-}$double-KO mice restores normal HSC function and lymphocyte development $(28,29)$. Moreover, deletion of the major proapoptotic p53 effector protein PUMA in $M y s m 1^{1^{-1}} \mathrm{Puma}^{-/-}$mice partially rescues hematopoiesis, restoring the viability of hematopoietic progenitors but not lymphocyte development (21). Overall, these studies established that p53 activation is the common pathway driving loss of HSC function and lymphopenia in Mysm1 deficiency. However, the mechanisms leading to p53 activation in Mysm1 deficiency have yet to be defined. In particular, the established molecular function of MYSM1 as a transcriptional activator and its essential role in p53 stress response repression remain difficult to reconcile.

To further characterize the functions of MYSM1 and the mechanisms leading to hematopoietic dysfunction in MYSM1 deficiency, we performed genome-wide analyses of MYSM1-regulated genes in hematopoietic stem and progenitor cells (HSPCs). This included RNA sequencing (RNA-Seq) of sorted Mysm1-deficient mouse HSCs and ChIP-Seq mapping of MYSM1 DNA binding sites in hematopoietic progenitor cell lines. We demonstrate a direct role for MYSM1 in the regulation of genes encoding protein components of the ribosome ( $R P$ genes) and other regulators of translation. Mechanistically, the dysregulation of $R P$ genes in Mysm1 deficiency was upstream of p53 activation and associated with reduced HSCs protein synthesis rates and p53-dependent anemia. We also describe a new patient with a homozygous nonsense variant in the MYSM1 gene, demonstrate reduction in protein synthesis rate and activation of p53 in patient blood cells, and compare disease presentation to ribosomopathy syndromes. Together, these findings indicate common mechanisms of hematopoietic dysfunction in MYSM1 deficiency and ribosomopathy disorders.

\section{Results}

Transcriptional landscape of Mysm1-deficient mouse HSCs. We previously established that MYSM1 has essential cell-intrinsic functions in the earliest hematopoietic compartments $(19,20)$, while MYSM1 loss in mature hematopoietic cells or the niche has minimal impact on hematopoiesis $(25,26,30)$. To gain an in-depth understanding of the mechanisms driving hematopoietic failure in Mysm1 deficiency, we performed RNASeq transcriptional profiling of FACS sorted primary HSC, MPP1, and MPP2 cells, gating on Lin ${ }^{-} \mathrm{CK}-$ $\mathrm{it}^{+} \mathrm{Sca}^{+}$(LSK) $\mathrm{CD}^{2} 50^{+} \mathrm{Flt3}^{-}$, and CD34-CD48- for HSCs, CD34+CD48- for MPP1, and CD $34^{+} \mathrm{CD} 48^{+}$ for MPP2 cells (Figures 1A and Supplemental Figure 1; supplemental material available online with this article; https://doi.org/10.1172/jci.insight.125690DS1) (31, 32). Cells were harvested from Mysm1 ${ }^{f l / f l}$ CreERT2 mice following tamoxifen-induced Mysm1 deletion and compared against cells from tamoxifen-treated Mysm $1^{f /+}$ CreERT2 and corn-oil vehicle-treated Mysm1 $1^{f l}$ animals. This inducible KO model focused our analysis on the direct effects of Mysm1 loss, eliminating many of the complex developmental phenotypes seen in constitutive Mysm1 deficiency (33). Cells from multiple mice were pooled to achieve yields of $>1 \mathrm{ng}$ 
of RNA per sample, and 3 independent samples were analyzed per genotype for each cell type for tamoxifen-induced mice and 2 independent samples for vehicle-treated mice.

Dimension reduction analysis of gene expression profiles showed a clear segregation based on the cell populations following their path of differentiation (PC1, 42.6\% variance) (Figure 1B). There was also a clear segregation based on genotype (PC2, 12.0\% variance), demonstrating a major transcriptome change in homozygous Mysm1-deficient cells (Mysm $1^{1 / \Delta}$ ) relative to the 2 control groups. Importantly, cells from the 2 control groups, the tamoxifen-treated $M y s m 1^{f l+}$ CreERT2 and vehicle-treated $M y s m 1^{f l}$ mice, clustered together, and this indicates that the cellular transcriptome is not affected by CreERT2 expression or tamoxifen treatment. For this reason, we grouped both control groups together as the WT group for subsequent analyses.

Differential gene expression analyses were conducted by comparing $M y s m 1^{\Delta / \Delta}$ with $M y s m 1^{W T}$ samples for each population. Across all comparisons, a total of 702 genes were considered significantly dysregulated, with a fold change $\geq 1.5$ and FDR $\leq 0.01$ (Supplemental Table 1). Mysm1 $1^{4 / 4}$ HSCs had the most profound dysregulation of gene expression, with 357 upregulated and 239 downregulated genes. Mysm $1^{4 / \Delta}$ MPPs showed fewer dysregulated genes (44 upregulated and 67 downregulated in MPP1, and 88 upregulated and 19 downregulated in MPP2), as well as lower average fold-changes in gene expression relative to the corresponding Mysm $1^{W T}$ cells (Figure 1C). This, along with high relative Mysm1 expression in HSCs (Supplemental Figure 2A), suggests that MYSM1 has more prominent cell-intrinsic roles as a transcriptional regulator in HSCs.

To explore the biological functions dysregulated within the transcriptome of $M y s m 1^{4 / \Delta}$ cells, we performed Gene Set Enrichment Analysis (GSEA) (34). GSEA highlighted upregulation of genes involved in cell proliferation, DNA replication, and mitosis in HSCs (Figure 1D and Supplemental Table 2), consistent with the previously reported loss of HSC quiescence in Mysm1 deficiency (20, 21). In contrast, MPP2-upregulated gene signatures were enriched for p53 pathway and apoptosis (Supplemental Table 2), reflecting the previously characterized activation of p53 stress responses in Mysm1-deficient hematopoietic progenitors $(21,28,29)$. Therefore, the transcriptome of Mysm1 deficiency showed differential signatures in HSCs and MPPs, which are in turn manifested in their corresponding distinct phenotypes. Importantly, our data demonstrate a highly substantial downregulation of genes involved in translation and ribosome biogenesis in $M y s m 1^{\Delta / \Delta}$ HSCs (Figure 1D).

Hierarchical clustering analysis segregated the 702 genes dysregulated in at least 1 cell type into Clusters I-IV (Figure 1E). Gene ontology (GO) enrichment analysis was performed for the genes in each cluster (Figure $1 \mathrm{~F}$ ). Detection of enriched biological processes was somewhat restricted for Clusters III and IV, due to the limited numbers of genes in these clusters. Nevertheless, Cluster III contained genes involved in apoptosis and cell cycle arrest, such as Cdkn1a, Ccnd1, P2rx7, Bcl2l1, and Zmat3, and it was upregulated in Mysm1 $1^{1 / 4} \mathrm{HSCs}$ and MPPs. Cluster IV contained MHC-II and other immunity-related genes and was upregulated in Mysm $1^{1 / \Delta}$ MPP2 cells in comparison with Mysm $1^{W T}$ MPP2 cells. Cluster II was highly enriched for genes involved in cell cycle progression that gradually increase in expression with the maturation of WT HSCs to MPP1 and MPP2 cells. In Mysm $1^{\Delta / \Delta}$ HSC and MPP1 cells, Cluster II genes were prematurely upregulated, concordant with the loss of quiescence and hyperproliferation of these cells in Mysm1 deficiency (20, 21). Importantly, Cluster I represented the genes reduced in expression in $M y s m 1^{1 / \Delta}$ cells, with the strongest reduction in $M y s m 1^{1 / \Delta} \mathrm{HSCs}$ (Figure 1C). Surprisingly, this cluster was enriched for genes involved in translation, including the eIF4A2 RNA helicase subunit of eIF4F cap-binding initiation complex, eEF1G involved in aminoacyl-tRNA delivery to the ribosomes, and others (Supplemental Table 1). The cluster also included 35 significantly downregulated genes encoding proteins of the small and large ribosomal subunits ( $R P$ genes).

MYSM1 maintains RP gene expression in HSCS. Given the strong association of $R P$ gene mutations to hematopoietic dysfunction in ribosomopathies including DBA (3), we performed further analyses focusing on the $R P$ gene set. GSEA comparing $R P$ gene expression in WT HSC, MPP1, and MPP2 cells revealed elevated $R P$ gene expression in HSCs relative to MPPs (Supplemental Figure 2, B and C). While this finding is unexpected due to the quiescent state, low protein synthesis, and distinct metabolic activities characteristic of HSCs $(1,2,35,36)$, it is consistent with previous reports (37). Further GSEA comparing $R P$ gene expression in our Mysm $1^{W T}$ and $M y s m 1^{\Delta / \Delta}$ data sets demonstrated a significant and global downregulation of $R P$ genes in $M y s m 1^{1 / \Delta}$ cells, with the most severe reduction in HSCs (Figure 2, A and B). We also performed GSEA focused specifically on the $R P$ gene set mutated in DBA syndrome and again showed prominent downregulation of these genes in $M y s m 1^{1 / 4}$ cells, with the most severe reduction in HSCs (Figure 2, C and D).

As the p53 stress response was previously established as a mediator of HSC dysfunction in Mysm1 deficiency $(28,29)$, we performed GSEA for p53-regulated stress response genes. We confirmed the upregulation 
A

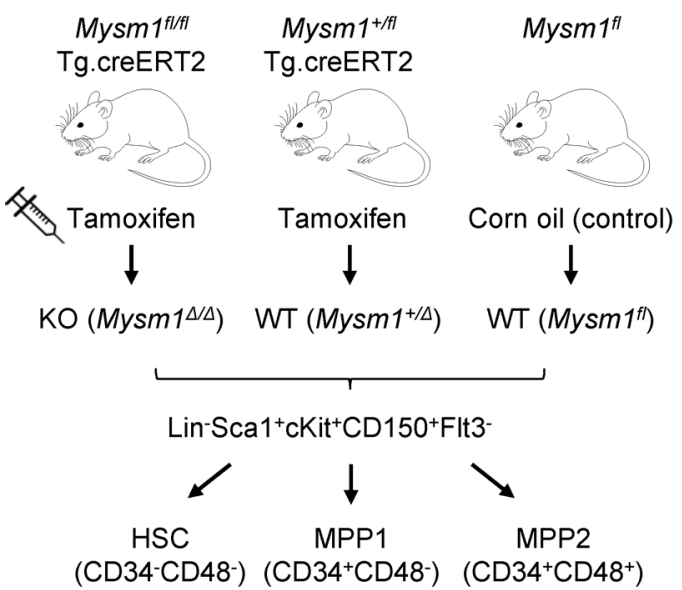

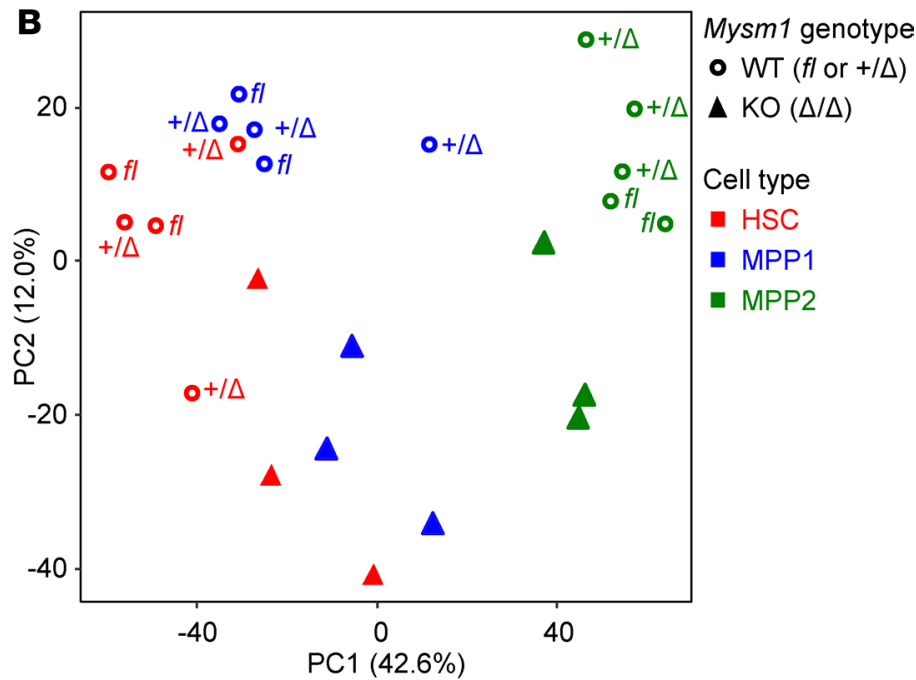

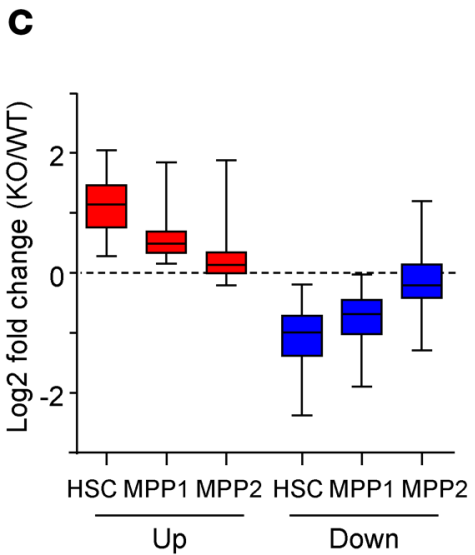

$\boldsymbol{F}$
D

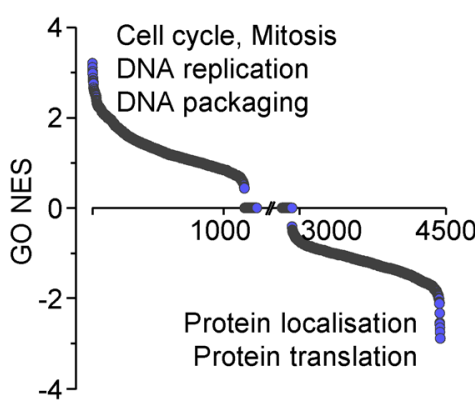

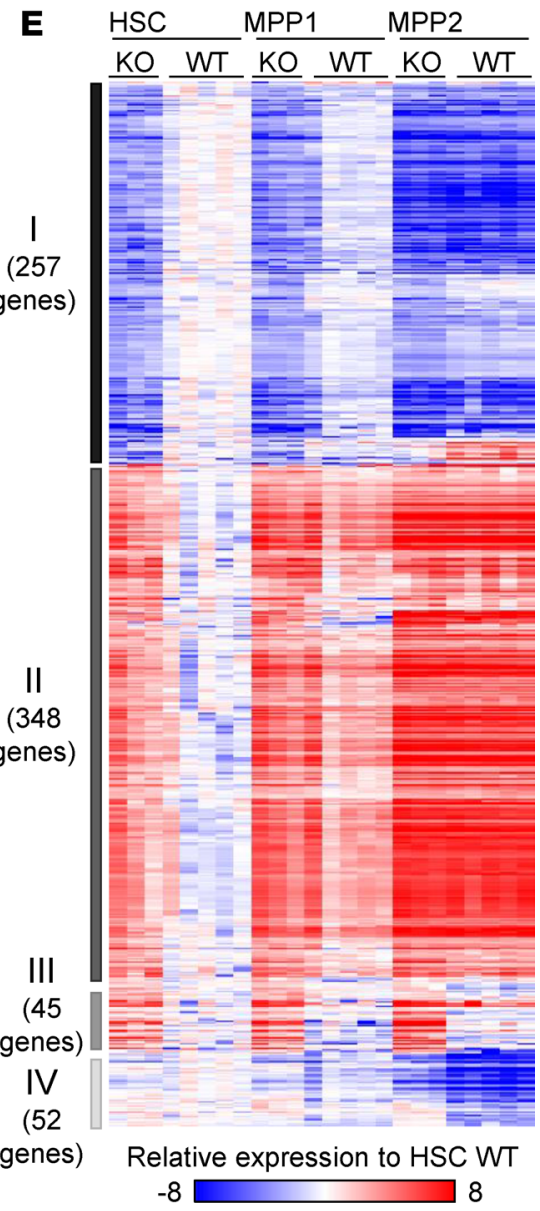

Figure 1. RNA-Seq analysis of the transcriptome of Mysm1-deficient HSC and MPP cells. (A) Schematic representation of the inducible Mysm1-deletion model in mice and the gating strategies for HSC, MPP1, and MPP2 cells. The KO group is composed of Mysm $7^{\text {flf }}$ CreERT2 mice following tamoxifen-induced Mysm1 deletion. The WT group is composed of tamoxifen-treated Mysm $7^{f /+}$ CreERT2 and corn-oil vehicle-treated Mysm $7^{f l}$ mice. (B) Partial Least Square Regression graph demonstrates the gene expression profiles of each RNA-Seq sample: differences between cell types are described by principal component 1 (PC1, 42.6\% variability) and differences between genotypes by PC2 (12\% variability). (C) Fold changes of 702 significantly dysregulated genes in Mysm1-deficient HSCs and MPPs. (D) Normalized enrichment scores (NES) of 4437 preestablished biological processes expression signatures used in the Gene Set Enrichment Analysis. (E) Heatmap displaying 702 significantly dysregulated genes when comparing KO with WT expression levels. The significance threshold is fold change $\geq 1.5$ and FDR $\leq 0.01$. Relative expressions to the average of HSC WT group are used to generate the heatmap. Hierarchical Clustering $(\mathrm{HCL})$ of the genes is performed, using Pearson correlation and average linkage, to generate the 4 gene clusters. (F) Gene ontology (GO) enrichment analysis on genes from the 4 clusters described in E. Top 4 enriched biological processes terms are displayed. 
of p53 target genes in Mysm1 $1^{4 / 4}$ HSCs and MPPs, but we observed that their induction was progressive, with the weakest effects in HSCs and strongest in MPP2 cells (Supplemental Figure 2D). We note that the higher expression of Mysm1 in HSCs relative to MPPs (Supplemental Figure 2A) coincides with a more prominent downregulation of $R P$ genes in $M y s m 1^{4 / 4}$ HSCs, which suggests a direct functional link between MYSM1 and $R P$ gene expression. In contrast, the progressive upregulation of $\mathrm{p} 53$ target genes in MPP2 cells suggests that their induction is an indirect response to the cellular stress of ribosomal dysfunction in HSCs.

In summary, our analyses of Mysm1-deficient HSC and MPP transcriptomes characterize the gene expression signatures underlying the loss of quiescence $(20,21)$ and p53 stress response activation $(21,28$, 29 ) in these cells, respectively. Additionally, we report a profound downregulation of $R P$ genes and genes encoding regulators of translation in Mysm1-deficient HSCs.

MYSM1 binding to RP gene promoters. To gain further insight into the genomic regions directly regulated by MYSM1 in hematopoietic cells, we mapped MYSM1 DNA binding sites by ChIP-Seq. The experiments were carried out in 2 murine hematopoietic progenitor lines: a multipotent progenitor line HPC7 (38) widely used as a model to study transcriptional programs of hematopoiesis $(39,40)$ and pro-B cell line Ba/F3 that was used in our previous work to model p53 activation and other molecular mechanisms of Mysm1 deficiency (21). The ChIP-Seq data reveal that MYSM1 binds at a relatively limited number of sites across the genome, corresponding to 2099 significant binding peaks, and the most MYSM1 binding sites are common between HPC7 and Ba/F3 cells (Figure 3A). Ordering the MYSM1 binding sites by their distance to the nearest gene transcription start site (TSS), the 2099 sites were classified as either gene-proximal $(<1 \mathrm{~kb}$ to TSS, 102 sites) or gene-distal (>1 kb to TSS, 1997 sites) (Figure 3A).

ChIP-Seq was carried out to characterize the epigenetic status at MYSM1-bound sites in HPC7 and $\mathrm{Ba} / \mathrm{F} 3$ cells. We assessed H3K27ac, a marker of active promoters and enhancers, and H2AK119ub, a repressive marker previously established as a target of MYSM1 in specific contexts (14). Gene-distal MYSM1 sites harbored the epigenetic status of transcriptionally inactive sites, with absence of H3K27ac and higher level of H2AK119ub (Figure 3A and Supplemental Figure 3). Conversely, the data reveal high levels of H3K27ac and low levels of H2AK119ub at the gene-proximal MYSM1 binding sites, indicating that they represent transcriptionally active gene promoters (Figure $3 \mathrm{~A}$ and Supplemental Figure 3).

To gain functional insight into MYSM1 binding targets, GO and disease ontology analyses were performed on genes in the vicinity of each MYSM1 binding site using Genomic Regions Enrichment of Annotations Tool (GREAT) (41). Genes near the 102 gene-proximal MYSM1 binding sites were highly enriched for biological process ontology terms "translation," "protein metabolic process," and "ribosome biogenesis" (Figure 3B) and disease ontology terms "congenital hypoplastic anemia" and "Diamond-Blackfan anemia" (Figure 3C). Importantly, all of these GO-terms refer to the same set of genes encoding ribosomal proteins, translation factors, and other functionally related proteins. Analysis of genes near the 1997 gene distal MYSM1 binding sites showed no significant enrichment of either biological process or disease ontology terms, highlighting the challenge of inferring a role for intergenic MYSM1 binding in gene regulation. Overall, these data provide further supporting evidence for a direct role of MYSM1 in regulating genes involved in ribosome assembly and translation.

Direct role of MYSM1 in the maintenance of $R P$ gene expression. To identify genes that are likely to be directly regulated by MYSM1 in HSCs and MPPs, we consolidated our RNA-Seq and ChIP-Seq data sets, searching for genes dysregulated in Mysm1 deficiency with nearby MYSM1 binding sites. Dysregulated genes in RNA-Seq Clusters II, III, and IV - related to regulation of cell cycle progression, apoptosis, and immunity (Figure 1, E and F) — had neither proximal nor distal MYSM1 binding sites (Figure 4A). This suggests that their expression is not directly controlled by MYSM1, but rather becomes dysregulated in Mysm1 deficiency through indirect mechanisms. In contrast, many of the downregulated genes in RNA-Seq Cluster I, including the genes encoding RPs and translational regulators, had MYSM1 binding sites within $1 \mathrm{~kb}$ of their TSS (Figure 4A and Supplemental Table 3), suggesting direct activation of their expression by MYSM1. Strikingly, 35\% of gene-proximal MYSM1 binding sites mapped to the promoters of Cluster I genes. Furthermore, 89\% of the genes that had MYSM1 binding sites in the ChIP-Seq data and were dysregulated in expression in $M y s m 1^{1 / 4}$ cells in RNA-Seq data-encoded ribosomal proteins and translation factors (Supplemental Table 3). The promoters of these genes were marked with high levels of histone H3K27ac and low levels of histone H2AK119ub (Figure 4B and Supplemental Figure 4), indicating their active transcription in HSPCs. 
A

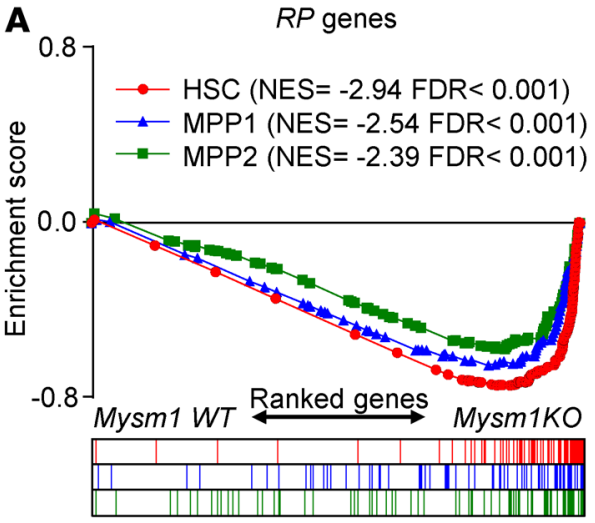

C

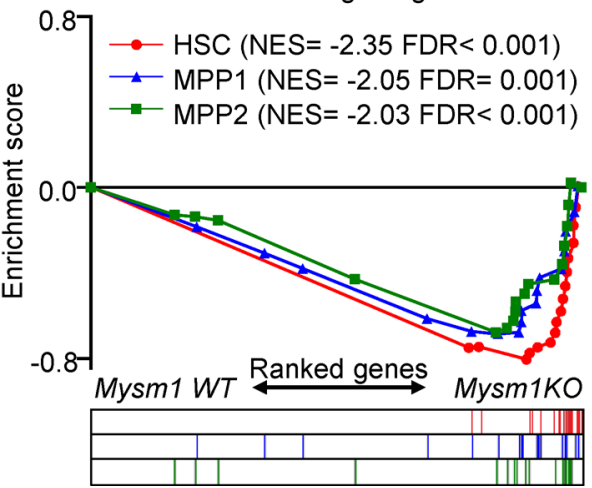

B
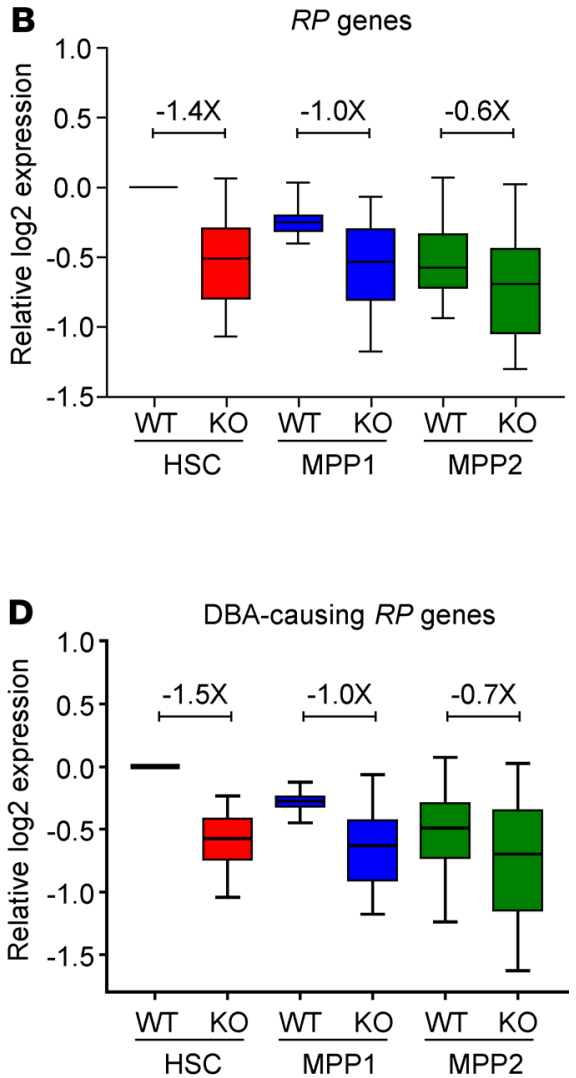

Figure 2. Ribosomal protein genes and DBA-causing genes have reduced expression in Mysm1-deficient HSC and MPP cells. (A and C) Gene Set Enrichment Analysis (CSEA) ranked and demonstrated reduced expression of 80 ribosomal protein $(R P)$ genes (A) 17 DBA-causing $R P$ genes in Mysm1-deficient cells (C) among 10,769 genes detected in HSCs and MPPs in the RNA-Seq data set. Each relevant gene is represented by a dot and a vertical bar below. (B and $\mathbf{D})$ Box plots showing reduced expression of $R P$ genes (B) and DBA-causing $R P$ genes (D) in Mysm1-deficient HSCs and MPPs.

A subset of the genes identified as directly regulated by MYSM1 was selected for further validation. This included 8 genes encoding proteins of the small and large ribosomal subunits, of which 3 are known to be mutated in DBA (Rps10, Rps24, Rpl11) (3), as well as the gene encoding translation factor eEF1G (Figure 4B). MYSM1 binding to the promoters of all the selected genes was validated through ChIP-quantitative PCR (ChIP-qPCR) in Ba/F3 lines (Figure 4C). Furthermore, the effect of MYSM1 loss on the expression of these genes was analyzed by qPCR, comparing previously established Mysm1-knockdown (shMysm1) $\mathrm{Ba} / \mathrm{F} 3$ cells against control cells expressing off-target shRNA against firefly luciferase (shFF) (21). This demonstrated downregulation in the expression of the genes in shMysm1 Ba/F3 cells (Figure 4D), consistent with our Mysm1 ${ }^{\Delta / \Delta}$ HSC transcriptome data. Therefore, we have validated MYSM1 binding to the promoters of these genes and have shown that MYSM1 loss results in their downregulation in vitro and in vivo, demonstrating the direct role of MYSM1 in their transcriptional regulation.

To further validate our ChIP-Seq findings, levels of histone modifications at promoters of MYSM1-regulated $R P$ genes were analyzed by ChIP-qPCR. We compared histone mark enrichment in knockdown shMysm1 and control shFF Ba/F3 cells, with a focus on DBA-mutated genes Rps10, Rps24, and $R$ pl11. Consistent with the active chromatin state observed in our ChIP-Seq data (Supplemental Figure 3) and with the high levels of $R P$ gene expression, levels of H2AK119ub at $R P$ gene promoters in both shMysm1 and shFF cells were very low. No significant increase in H2AK119ub levels was seen in the Mysm1-knockdown Ba/F3 cells (data not shown), suggesting that MYSM1 promotes the expression of these genes through mechanisms other than direct catalytic activity on H2AK119ub. This is consistent with the classical role of histone H2AK119ub as a marker of stable long-term gene silencing during cell differentiation and development, but not in the regulation of housekeeping genes such 
A

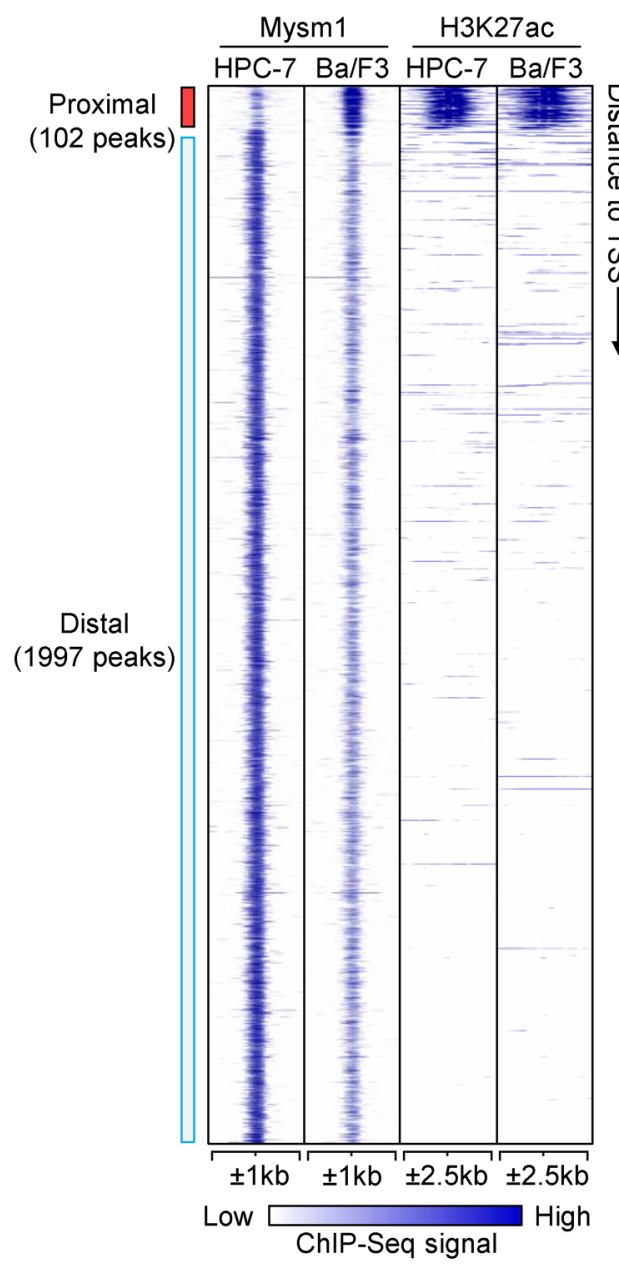

B

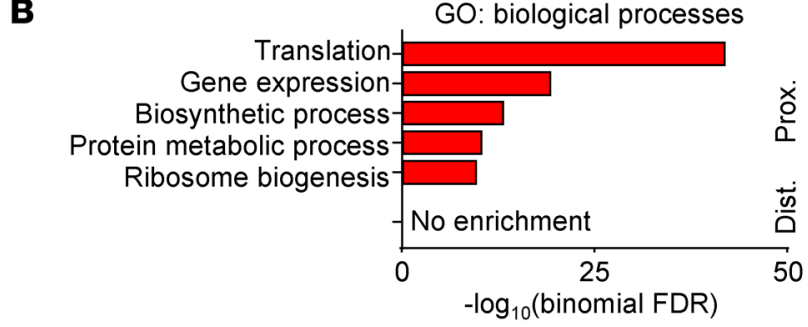

Figure 3. ChIP-Seq reveals MYSM1 binding at ribosomal protein gene promoters. (A) Heatmap showing the intensities of MYSM1 and H3K27ac around 2099 MYSM1 binding sites identified in ChIP-Seq data from HPC7 and $\mathrm{Ba} / \mathrm{F} 3$ cells. The sites are ranked based on their distances to the nearest gene TSS. The gene-proximal sites are defined as having the nearest gene TSS within $1 \mathrm{~kb}$. The gene-distal sites are defined as having the nearest gene TSS farther than $1 \mathrm{~kb}$. (B and C) Gene Ontology (B) and disease ontology (C) analyses on the nearest genes to each MYSM1 binding site, performed on the GREAT website (http://great.stanford. edu). The $-\log _{10}$ (binomial FDR) value for each term is plotted.

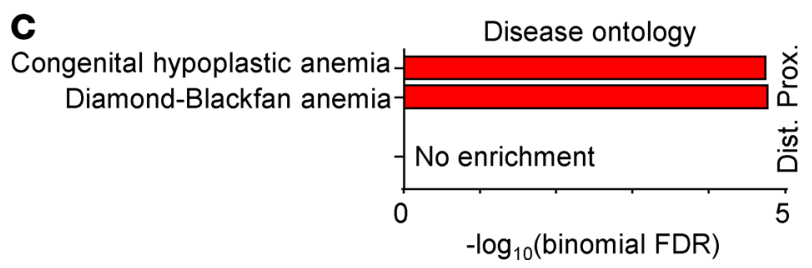

as $R P$ genes (42-44). On the other hand, we observed a consistent reduction in the levels of histone $\mathrm{H} 3 \mathrm{~K} 27 \mathrm{ac}$ at all $3 R P$ gene promoters tested in Mysm1-knockdown Ba/F3 cells, correlating with and suggesting a mechanistic link to their reduced gene expression (Figure 4E).

Protein synthesis rates are diminished in Mysm1-deficient HSCs. Since ribosome function and protein synthesis are dependent on the coordinated expression of $R P$ genes, we sought to assess the effects of $R P$ gene downregulation on ribosome function in Mysm1-deficient hematopoietic cells. Knockdown shMysm1 and control shFF Ba/F3 cells were treated with an inhibitor of rRNA synthesis (actinomycin D) or an inhibitor of protein translation (cycloheximide). Over a range of concentrations of both inhibitors, Mysm1-deficient Ba/F3 cells showed increased levels of cell death as compared with control cells (Figure 5, A and B). This indicated that loss of MYSM1 sensitizes hematopoietic progenitors to inhibition of ribosome biogenesis and protein synthesis. 
A

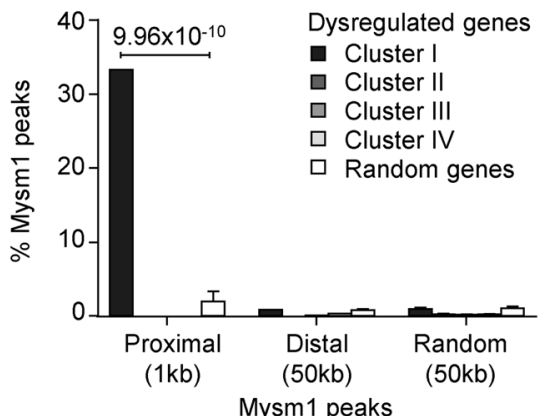

C

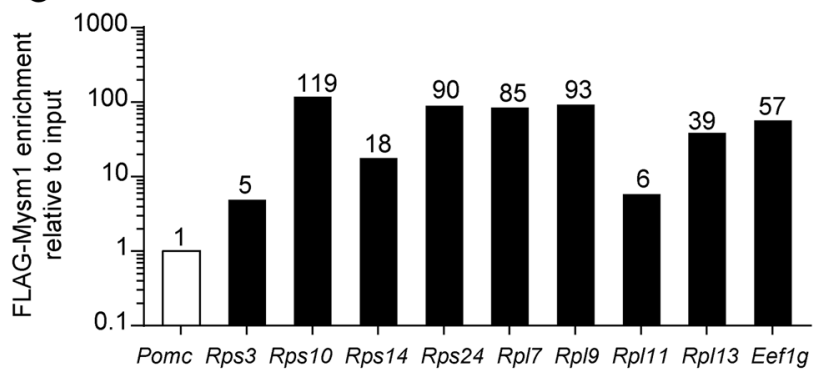

B

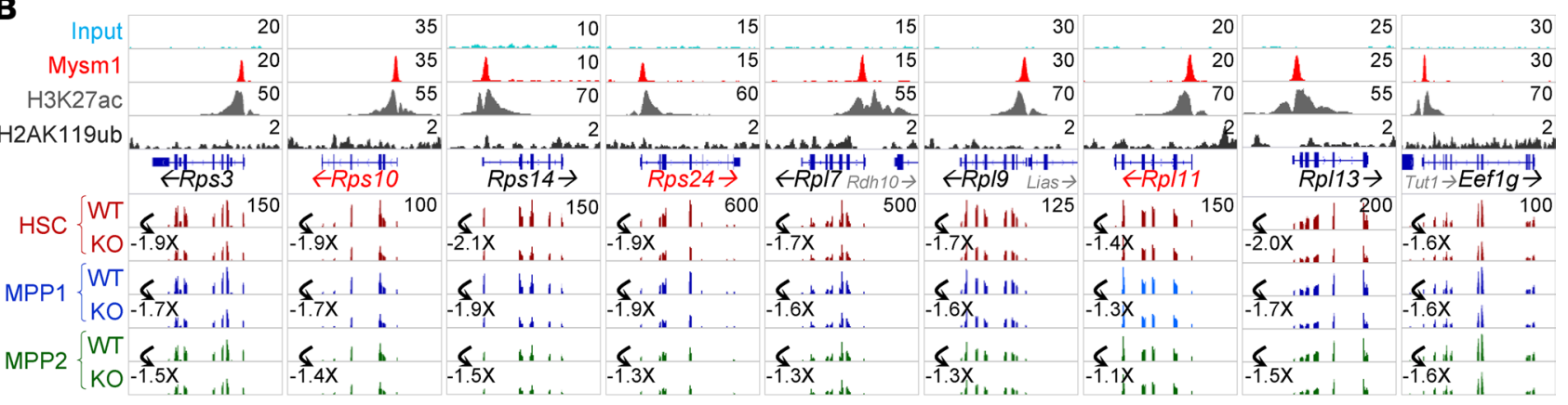
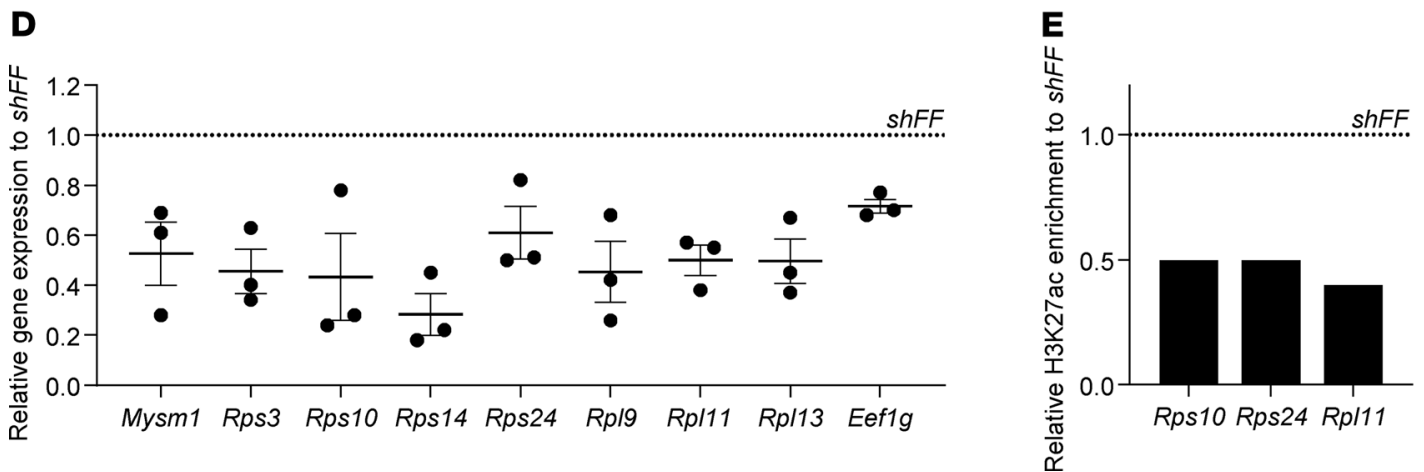

Figure 4. Validation of the direct role of MYSM1 in the regulation of ribosomal protein gene expression. (A) Consolidation of ChIP-Seq and RNA-Seq data sets showing that gene-proximal MYSM1 binding sites preferentially locate near Cluster I genes dysregulated in Mysm1-deficient HSC/MPP cells. The percentages of MYSM1 binding sites that have at least 1 significantly dysregulated gene TSS within the indicated search window are plotted. The random genes cluster consists of 10 groups of 300 genes randomly selected from 10,169 expressed genes. The random binding sites consist of 10 groups of 2000 genomic locations randomly selected from the $\mathrm{mm} 9$ genome. A binding site with more than 1 gene TSS from the same gene cluster is counted only once. Fisher Exact Test is used to calculate the $P$ value. (B) Genomic snapshots of select dysregulated genes. ChIP-Seq tracks of input DNA, MYSM1, H3K27ac, and H2AK119ub are shown on the top 4 lanes. The gene feature track is shown in the middle. Averaged RNA-Seq tracks are in the bottom 6 lanes, with fold changes comparing expression levels in WT and KO samples indicated for each cell type. The maximum data range of each track is indicated at the top right corner of the track. (C) Enrichment of MYSM1 at RP gene promoter sites, validated with ChIP-qPCRs in MYSM1FLAG Ba/F3 hematopoietic progenitor cells. Data presented is from 1 experiment and were reproduced in 3 independent experiments. (D) Downregulation of $R P$ gene expression in knockdown shMysm1 Ba/F3 cells relative to control shFF Ba/F3 cells, validated with qPCRs. Each dot represents relative fold change from 1 of 3 independent experiments. Two to 3 technical replicates were performed per experiment. (E) Representative H3K27ac ChIP-qPCRs showing reduced relative enrichments in knockdown shMysm1 Ba/F3 cells relative to control shFF Ba/F3 cells. Data presented are from 1 experiment and were reproduced in 2 independent experiments.

We further analyzed the effects of Mysm1 deficiency on protein synthesis rates of mouse primary HSCs in vivo, using the O-propargyl-puromycin (OPP) incorporation method $(1,45)$. OPP was administered i.p. to $M y s m 1^{-1-}$ and WT mice, and their BM was harvested 1 hour later for flow cytometric analysis of intracellular OPP. We observed a significant reduction of OPP incorporation in Mysm $1^{-1-}$ HSCs, indicating reduced protein synthesis rates (Figure 5, C and D, and Supplemental Figure 5). This provides further supporting evidence for the functional significance of $R P$ gene downregulation as a mediator of hematopoietic dysfunction in Mysm1 deficiency. 
Downregulation of RP gene expression in Mysm1 deficiency is independent of p53 activation. Activation of $\mathrm{p} 53$ stress responses and induction of its downstream proapoptotic effector PUMA are some of the defining characteristics of $M y s m 1^{-/-}$hematopoietic cells and a driving mechanism for Mysm $1^{-/-}$phenotypes (21,

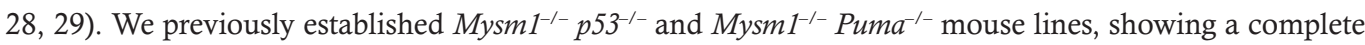
rescue of HSC functions and lymphocyte development with $p 53$ deletion and a partial rescue of progenitor cell numbers with Puma deletion $(28,29)$. Here, we further used these double-KO lines to dissect the relationship between p53/PUMA pathway activation and $R P$ gene downregulation in Mysm1 deficiency.

We first performed RNA-Seq on the combined HSC/MPP1/MPP2 cell population from Mysm1 $^{-1}$ Puma $^{-/}$, Mysm1 $^{-/-}$Puma $^{+/-}$, Mysm1 $^{+/+}$Puma $^{-/}$, and WT mice, gating on LSK and CD $150^{+}$. The data were analyzed together with our previously published RNA-Seq data sets from MPP3/MPP4 (LSK CD150-) cells from the same mice (Supplemental Figure 6 and Supplemental Table 4) (21). A dimension reduction analysis (Supplemental Figure 6A) confirmed clear separation between the gene expression profiles of CD150 ${ }^{+}$and CD150- cells (PC1, 41.7\%) and between Mysm1-deficient and Mysm1 WT samples (PC2,

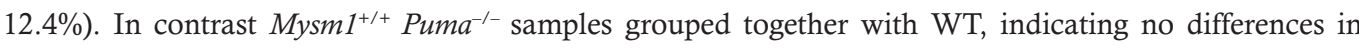
their gene expression profiles (Supplemental Figure 6A). Unbiased clustering of the genes dysregulated in Mysm1-deficient samples was performed, resulting in 8 clusters of gene expression patterns (Clusters 1-8; Supplemental Figure 6B). Consistent with our previous findings (21), activation of p53 stress response was a major signature of Mysm1 deficiency that persisted in $M y s m 1^{-1-} P_{m m a^{-1}}$ samples, with Cluster 1 containing hallmark p53 target genes, such as Cdkn1a/p21, Bax, and Pmaip1/NOXA (Supplemental Figure 6B and Supplemental Table 4). Importantly, downregulation of $R P$ gene expression in Mysm1 deficiency was also

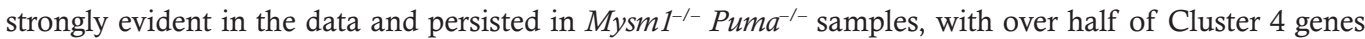
encoding RPs, eEF1G, and other functionally related proteins (Supplemental Figure 6B and Supplemental Table 4). GSEA analyses further confirmed a global and significant downregulation of $R P$ gene expression in $M y s 1^{-1-}$ HSPCs, including both LSK CD150 ${ }^{+}$and LSK CD150- subsets. Importantly, this significant downregulation of $R P$ gene expression was found to persist in $\mathrm{Mysm}^{-1-} \mathrm{Puma}^{-/-} \mathrm{HSPC}$ (Figure 6A and Supplemental Figure 6C), demonstrating that it is independent of PUMA and the downstream apoptosis of Mysm1-deficient HSPCs.

We then assessed the expression of $R P$ genes in $M y s m 1^{-1-} p 53^{-1-}$ double-KO mice, performing qPCR on sorted LSK CD150- hematopoietic progenitors. The data once again show a persistent downregulation in the expression of RP genes and the translation factor eEF1G in $M y s m 1^{-/-} p 53^{-/-}$HSPCs (Figure 6B). Protein levels of the translation factor eEF1G were analyzed by intracellular flow cytometry, demonstrating an equivalent reduction in eEF1G levels in the HSPCs of both $M y s m 1^{-/-}$and $M y s m 1^{-/-} p 53^{-/-}$mice (Figure 6, C and D). The persisting dysregulation of $R P$ gene and Eeflg expression in $M y s m 1^{-1-} p 53^{-1-}$ HSPCs, despite a rescue of hematopoietic phenotypes, therefore demonstrates that these transcriptional changes are independent of p53 stress response activation, HSC loss of quiescence, and lymphopenia. These findings support a model where $R P$ gene dysregulation is the direct outcome of Mysm1 deficiency and is upstream and causative for p53 activation and hematopoietic phenotypes in Mysm1 deficiency.

Anemia phenotypes of Mysm1 deficiency are mediated by p53 stress response activation. Anemia is a major feature of human ribosomopathies, such as DBA $(8,46)$, where it is associated with p53 activation in the affected cells, in both human patients (11-13) and mouse models (47-49). Importantly, MYSM1 deficiency in both humans and mice is also associated with anemia (16-18), and we previously reported that Mysm1 ${ }^{-1}$ mice have reduced erythrocyte count, increased mean corpuscular volume (MCV) and mean corpuscular hemoglobin $(\mathrm{MCH})$, but normal $\mathrm{MCH}$ concentration (MCHC) (19). The mechanisms underlying this anemia have not been previously investigated.

We tested for the role of p53 as a mediator of anemia in $M y s m 1^{-1-}$ mice (Figure 7). Loss of p53 in Mysm $1^{-/-} p 53^{-/-}$mice resulted in a striking complete rescue of the $M y s m 1^{-1-}$ erythroid phenotype, with a full normalization of erythrocyte count in the blood (Figure 7A) and megakaryocyte-erythroid progenitor (MEP) numbers in the BM (Figure 7B), restoration of hematocrit and blood hemoglobin levels (Figure 7C), and normalization of $\mathrm{MCV}$ and MHC parameters (Figure 7D). We therefore conclude that p53 activation is the essential mediator of anemia in Mysm1 deficiency. Together with our previous report showing a complete rescue of lymphopenia in $M y s m 1^{-1-} p 53^{-1-}$ mice (28), this finding further demonstrates that p53 activation mediates the multi lineage depletion of mature hematopoietic cells in Mysm1 deficiency.

$B b c 3 /$ PUMA and Cdkn1a/p21 are p53-inducible factors that act as key mediators of p53-regulated apoptosis and cell cycle arrest, and they are strongly overexpressed in Mysm $1^{-1-}$ HSPCs (21). We observed 
A
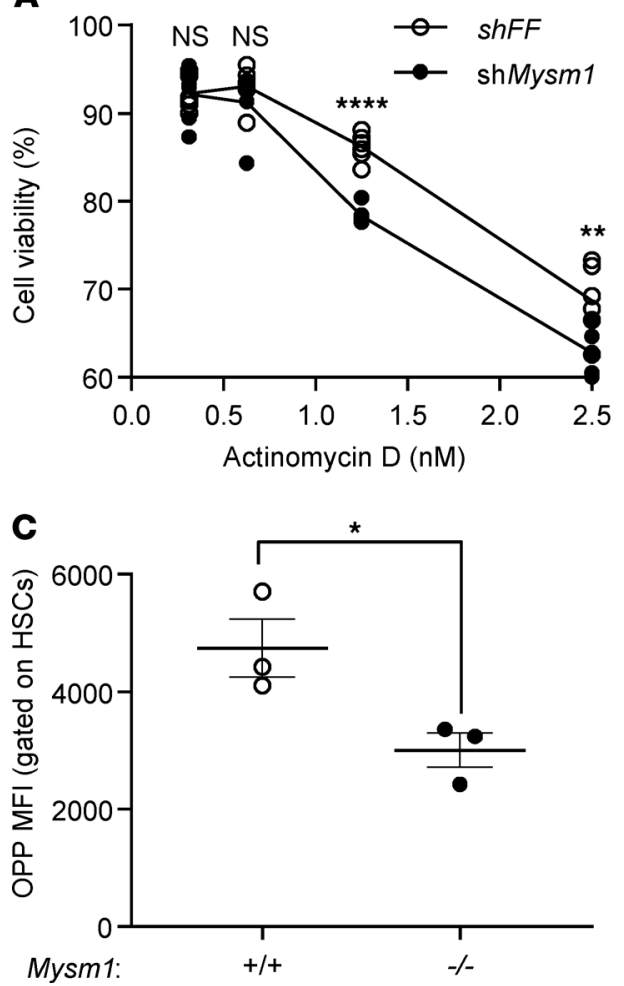

B

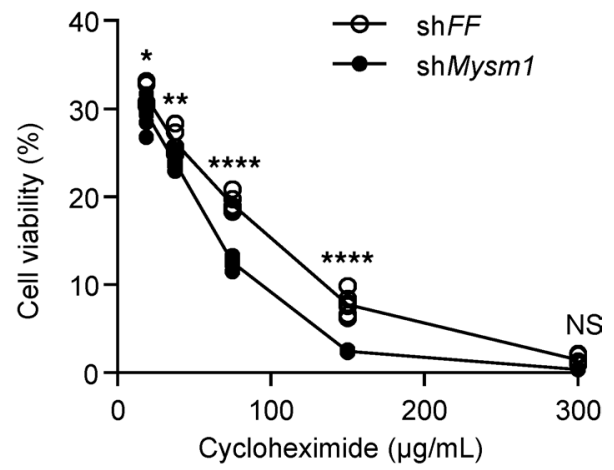

D

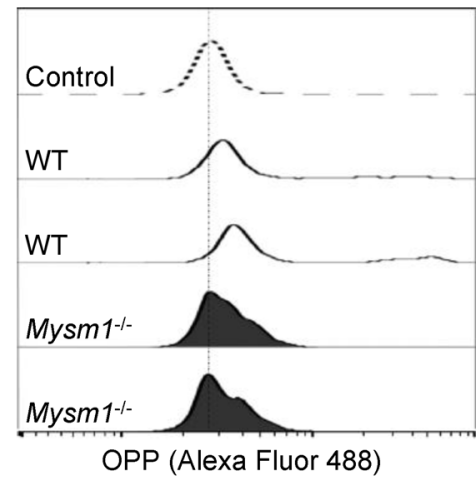

Figure 5. Hypersensitivity to ribosomal stress and reduction in protein synthesis rates in Mysm1 deficiency. (A and B) Mysm1-knockdown Ba/F3 cells are hypersensitive to ribosomal and translational stress. Control shFF and knockdown shMysm1 cells were treated with actinomycin D (A) or cycloheximide (B) across of a range of concentrations for 48 hours, and cell viability was measured by flow cytometry. The data are from 3 shMysm1 and 3 shFF lines, each analyzed in duplicate, and were reproduced in 2 independent experiments. Statistical comparisons were made using 2-way ANOVA with Holm-Šidák's post hoc test; ${ }^{*} P<0.05,{ }^{* *} P<0.01$, ${ }^{* * *} P<0.0001$. (C and $\left.\mathbf{D}\right)$ Reduction in the protein synthesis rate in $\mathrm{Mysm7}^{-/-}$primary HSCs, measured using the in vivo OPP-incorporation method. (C) Dot plot comparing the OPP incorporation levels in WT and Mys $\mathrm{m1}^{-1-}$ HSCs. Data presented are from 3 mice per genotype; the results were reproduced across 2 independent experiments; means \pm SEM are presented; statistical comparison using Student's $t$ test; ${ }^{*} P<0.05$. (D) Representative flow cytometry histograms showing OPP-incorporation in HSCs from 2 WT and 2

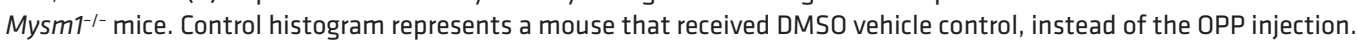

that the loss of Cdkn1a/p21 does not affect the $\mathrm{Mysm}^{-/-}$erythroid phenotype, with $M y s m 1^{-1-} p 21^{-/-}$mice being phenotypically equivalent to Mysm $1^{1^{-1}}$ in all parameters (Supplemental Figure 7). In contrast, loss of $B b c 3 /$ PUMA resulted in a partial rescue of the erythroid phenotype, with a mild but significant increase in erythrocyte count, hematocrit, and blood hemoglobin concentration in $M y s m 1^{-1-} \mathrm{Pum}^{-1-}$ as compared with Mysm $1^{-/-}$mice (Supplemental Figure 8). Considering our previous finding of persistent p53 activation in Mysm1 $1^{-1-}$ Puma $^{-1-}$ hematopoietic cells (Supplemental Figure 6 and Supplemental Table 4) (21), we conclude that the anemia in Mysm1 deficiency is triggered in part via p53/PUMA-mediated cell apoptosis, in addition to other p53-dependent but PUMA-independent mechanisms.

Overall, we demonstrate that MYSM1 directly regulates the expression of the genes encoding ribosomal proteins and translation regulators in HSCs. We propose that Mysm1 deficiency results in ribosomal stress that triggers p53-mediated anemia and other hematopoietic dysfunction. Given the many lines of evidence linking anemia and BM failure in DBA and other ribosomopathies with p53 activation (10-13, 47-50), our work highlights the common mechanisms underlying hematopoietic dysfunction in MYSM1 deficiency and ribosomopathy syndromes.

Clinical features and mechanisms of MYSM1 deficiency in humans. MYSM1 deficiency is a rare congenital disorder, with only 6 patients described previously $(16-18,51)$. Here, we characterize a 10-month old male patient with a potentially novel homozygous nonsense MYSM1 gene variant. The index patient is a fourth child of consanguineous parents (first cousins) from Turkey (Figure 8A). His parents and 2 older sisters are healthy. Of note, the parents' first child died at the age of 9 months due to a mitochondrial DNA depletion syndrome, 

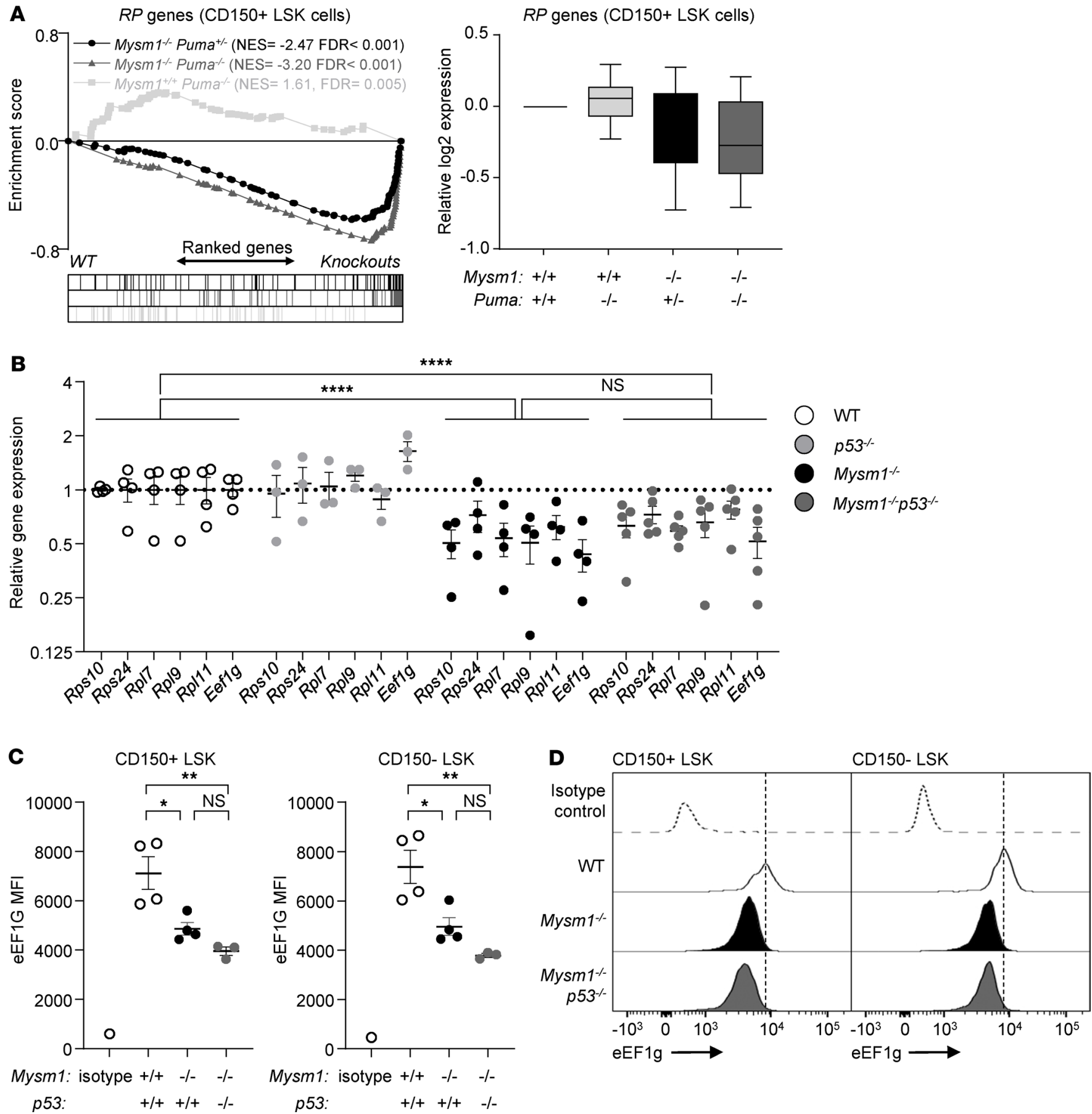

Figure 6. MYSM1-dependent regulation of ribosomal protein genes is independent of p53 activation. (A) Gene Set Enrichment Analysis (GSEA)

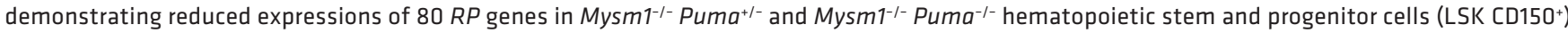
relative to control WT cells; 10,987 genes expressed in the RNA-Seq data set are ranked based on signal/noise ratio, and each relevant $R P$ gene is represented by a dot and a vertical bar below. Box plots for the relative $\log _{2}$ expression of the $R P$ genes also show downregulation in Mysm ${ }^{1 /-}$

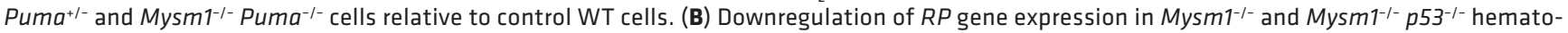
poietic progenitor cells; FACS-sorted LSK CD150- cells were analyzed by QPCR. Mean \pm SEM from 3-5 mice per group is presented; statistical comparisons using 2-way ANOVA; ${ }^{* * *} P<0.0001$. (C and $\left.\mathbf{D}\right)$ Analysis of eEF1G protein levels in hematopoietic stem and progenitor cells of WT, Mysm $1^{-1-}$, and Mys $\mathrm{m}^{-1-} \mathrm{p}^{-1 /-}$ mice by intracellular flow cytometry. Dot plots showing mean fluorescence intensity (MFI) of eEF1C staining (C), and representative

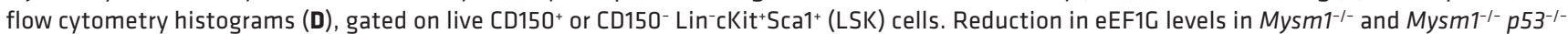
groups relative to the control WT group is shown. Mean \pm SEM from 3-4 mice per genotype is presented; statistical comparisons using 1-way ANOVA with Bonferroni's post hoc test; ${ }^{*} P<0.05,{ }^{* *} P<0.01$. 
A

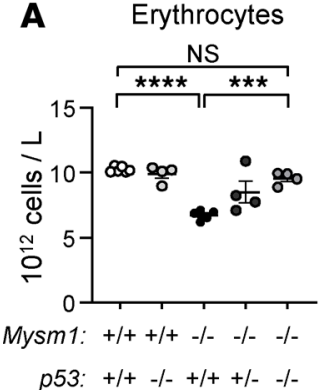

B
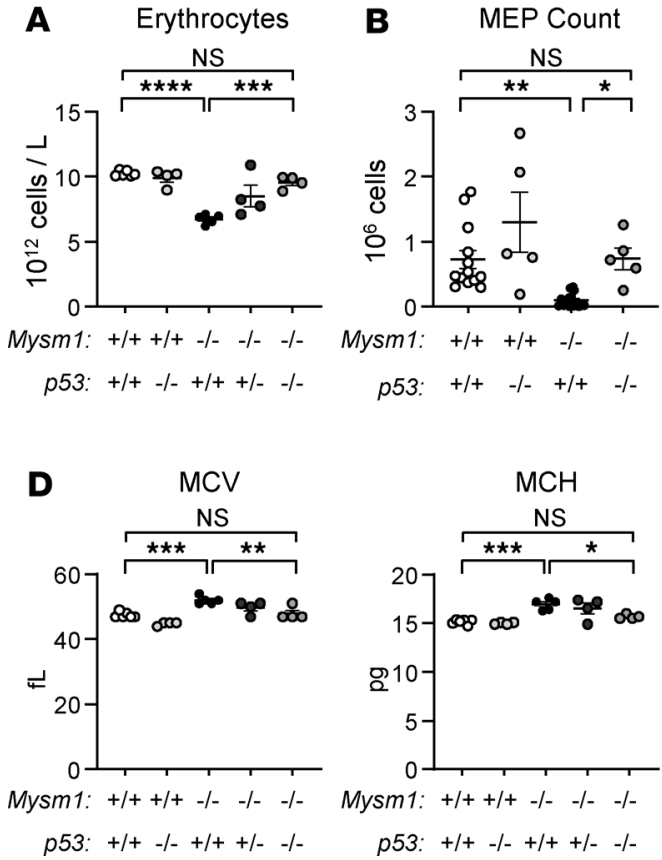

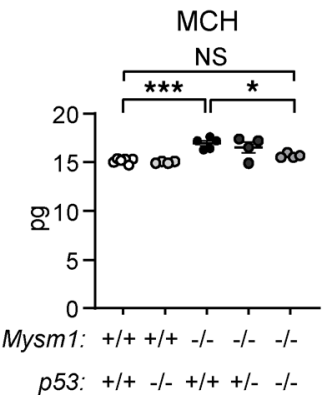

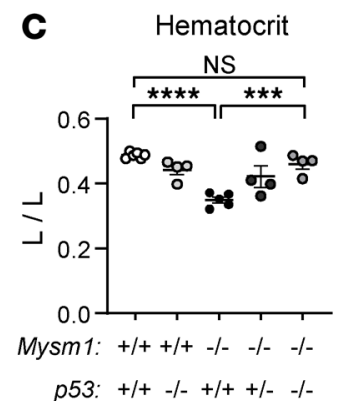

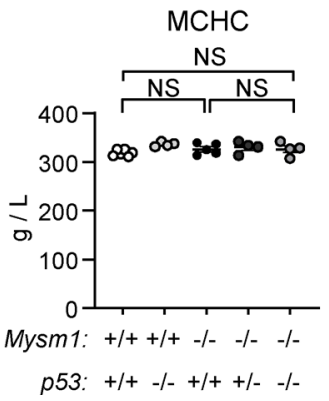

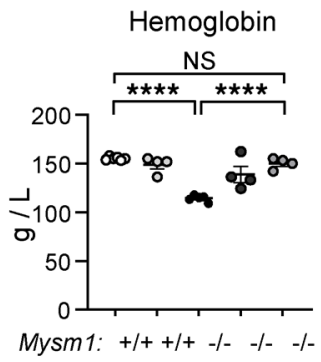

p53: $+/+-/-+/++/--/-$
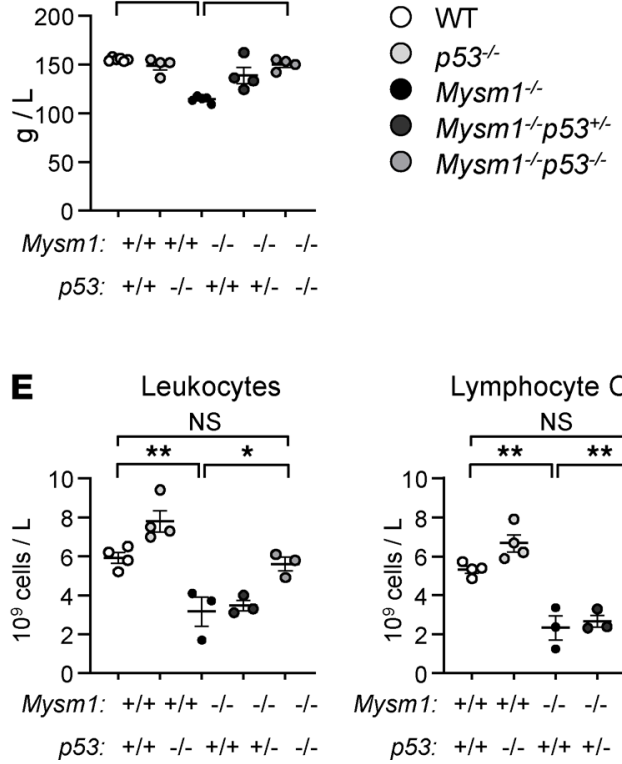

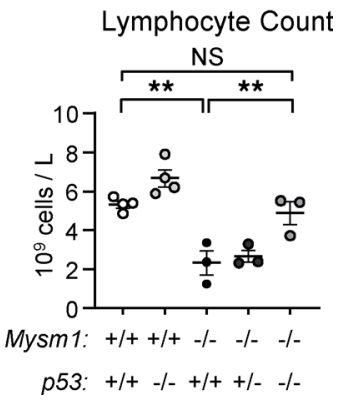

Figure 7. Loss of $\mathrm{p} 53$ rescues the anemia phenotype caused by Mysm1 deficiency. The data presented are from WT, $p 53^{-1-}, M_{y s m 1^{-1-}, M y s m 1^{-1-}} p 53^{+/-}$, and Mysm1 $1^{-1-} p 53^{-/-}$mice. (A) Hematology analysis of erythrocyte counts in peripheral blood of the mice. (B) MEP cell number in mouse BM, presented per 1 tibia and femur, gating on Lin-cKit ${ }^{+}$Sca1- CD34-CD16/32-CD127- cells. (C) Hematocrit and blood hemoglobin concentration. (D) MCV, MCH, and MCHC parameters. (E) Hematology analysis of leukocyte and lymphocyte counts in mouse blood. Mean \pm SEM from $\geq 3$ mice per genotype is presented; statistical comparisons using 1-way ANOVA with Bonferroni's post hoc test; ${ }^{*} P<0.05,{ }^{* *} P<0.01,{ }^{* *} P<0.001,{ }^{* * *} P<0.0001$.

caused by a homozygous 4-bp deletion in DGUOK; however, prenatal diagnosis from chorionic villous sampling in the index patient showed that he is not homozygous for the 4-bp DGUOK deletion. Additional conventional chromosome analysis showed male karyotype (46, XY karyotype) without pathological findings.

Birth took place at 38th week of gestation by cesarean section. Postpartum, anemia (hemoglobin [Hb] $8.1 \mathrm{~g} / \mathrm{dL}$ ), and leukopenia were noticed, and at the age of 3 weeks, RBC transfusion was required (Hb 6.8 $\mathrm{g} / \mathrm{dL}$ ) (Figure 8C). Longitudinal hematologic profile of the patient is provided in Supplemental Table 5A, and a comparison of the disease presentation to the diagnostic criteria of major ribosomopathy syndromes is provided in Supplemental Table 6. The main hematologic findings were transfusion-dependent hyporegenerative anemia with normal $\mathrm{MCH}$ and $\mathrm{MCV}$, mild to severe neutropenia with regeneration during infection, and mild BM cytopenia. Mild thrombocytopenia was observed only once ( $95 \mathrm{G} / \mathrm{L})$, and monocytopenia developed over time (Supplemental Table 5A). Flow cytometry of peripheral blood demonstrated reduction in neutrophils, basophils, monocytes, and B cells (Figure 8D and Supplemental Table 7). Hypogammaglobulinemia ( $\mathrm{IgG}<108 \mathrm{mg} / \mathrm{dL}$ ) was seen at the age of 11 months. The patient also had significantly elevated erythrocyte adenosine deaminase (eADA) (1366 IU/L EC, $n=250-650$ ), characteristically seen in DBA syndrome (52). Further routine diagnostics did not identify the cause for the symptoms, with normal $\mathrm{Hb}$ electrophoresis and exclusion of folic acid, vitamin B12, or iron deficiency. Moreover, the patient provided no evidence of pancreas insufficiency (normal elastase excretion in feces), characteristically seen in SDS (4). Discrete dysmorphic features were present, including midface hypoplasia, brachydactyly, and rhizomelic shortening of arms. A mild retardation of motor development was diagnosed at the age of 7 months. Genetic counseling took place at the age of 10 weeks, and genetic diagnostics were initiated. Meanwhile, the patient had repetitive need for transfusions, was given antibiotic prophylaxis, and has been listed for HLAmatched family donor HSC transplantation, as successfully performed earlier for MYSM1 deficiency (18).

Exome sequencing identified a homozygous nonsense variant, leading to premature stop codon, in MYSM1 exon 8 (c.869C > G, p.Ser290*), that was further validated by targeted Sanger sequencing (Figure $8 B)$. No mutations were identified in known DBA-causing genes (3), and complete information about rare and novel nonsynonymous homozygous variants identified in the patient's exome sequencing data is provided in Supplemental Table 5B. Importantly, MYSM1 was the only affected gene in this patient that was 
A

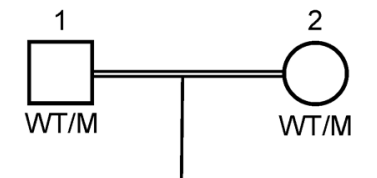

I

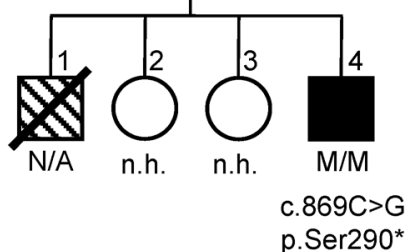

B

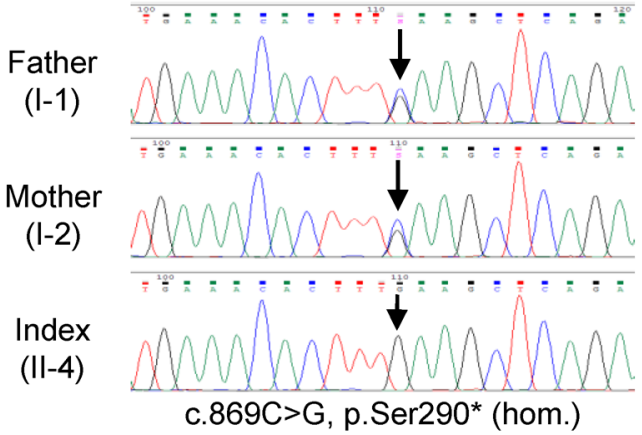

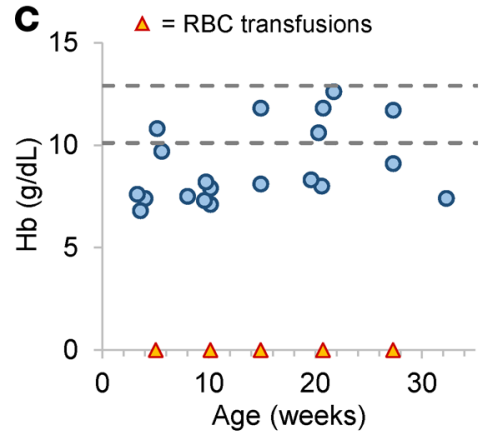
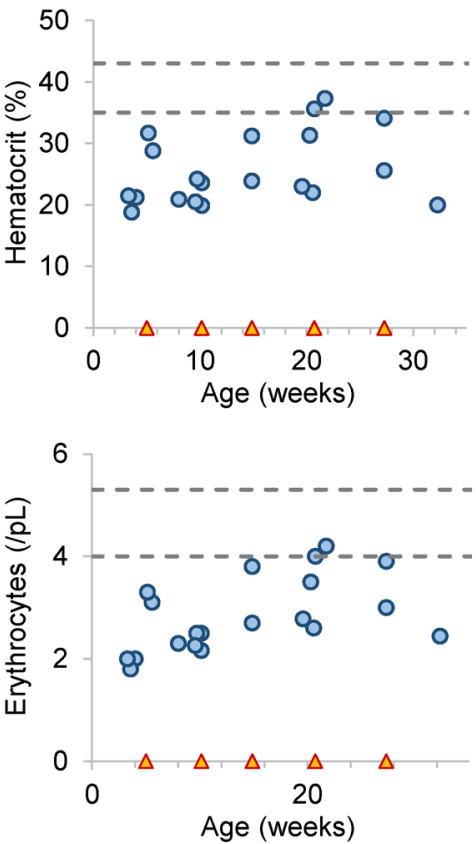
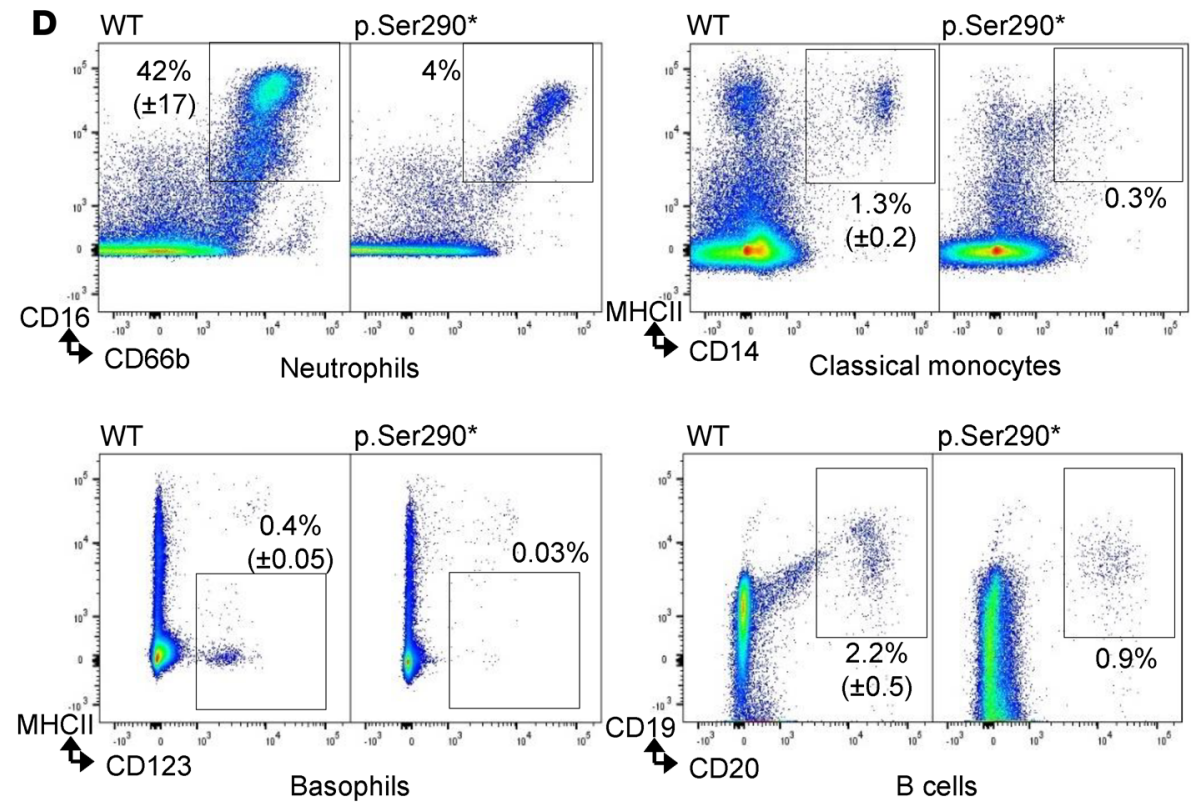

WT

Classical monocytes

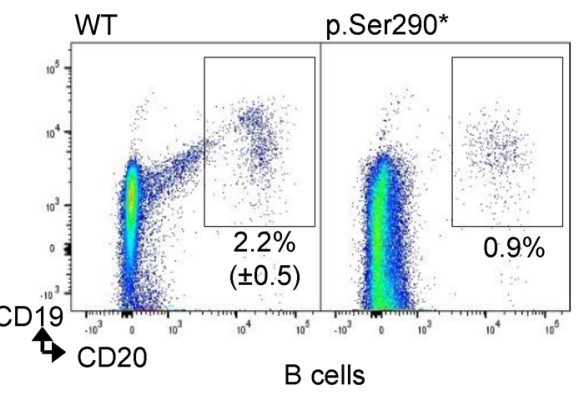

E

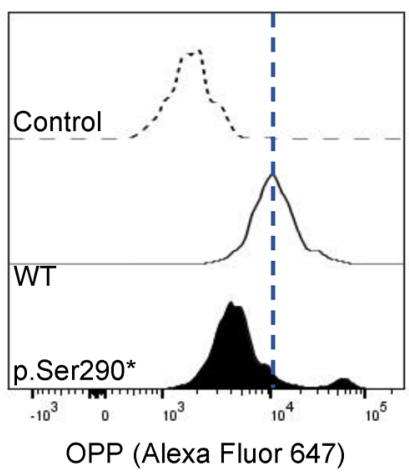

$\mathbf{F}$

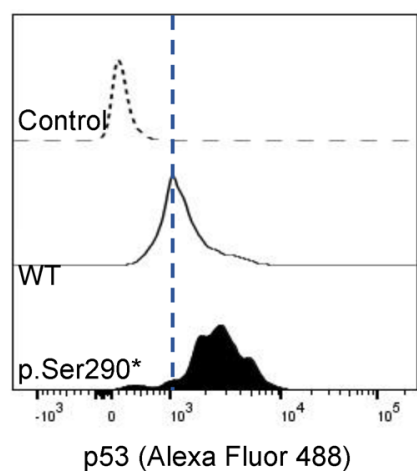

Figure 8. Characterization of the patient with homozygous nonsense mutation in MYSM1 gene. (A) Family pedigree with segregation of MYSM1 variant. Index patient (II-4) with transfusion dependent anemia was born from a consanguinous union; M/M, homozygous carrier; WT/M, heterozygous carrier; n.h., not homozygous; diagonal line, deceased individual. The deceased brother (died at the age of 9 months due to a mitochondrial DNA depletion syndrome) was not available (N/A) for testing. (B) Sanger sequence chromatograms of detected variant C.869C>C (NM_001085487.2) in MYSM1; homozygous in index patient, heterozygous in each parent. (C) Longitudinal hematological assessments of the patient, demonstrating anemia phenotype associated with reduced hemoglobin ( $\mathrm{Hb}$ ), hematocrit, and erythrocytes. Timing of erythrocyte transfusions is indicated and is the likely cause of the variations in the data. (D) Reduced frequency of neutrophils

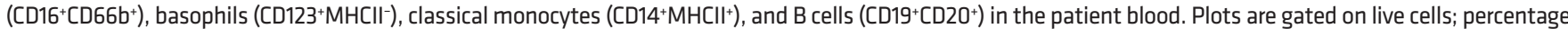
of cells within each gate is shown with the control figure representing mean \pm SD of 3 independent biological samples and the patient figure representing an average of 3 technical replicates from a single blood sample. Full data on all blood cell types in provided in Supplemental Table 7. (E and F) Representative flow cytometry histograms showing reduction in protein synthesis rate and increase in $\mathrm{p} 53$ protein level in the index patient; the plots are gated on Lin- ${ }^{-} \mathrm{CD} 38^{+} \mathrm{CD} 34^{-}$ hematopoietic progenitor cells, with full data on all blood cell populations provided in Supplemental Table 8. Protein synthesis rate is measured using the 0-propargyl-puromycin (OPP) incorporation method; control samples represent OPP-untreated but stained cells or cells stained with an isotype-control antibody. 
previously linked to anemia and leukopenia in human patients and mouse models (16-19), convincingly pointing to MYSM1 as the major candidate causative gene for the pathologies. Both parents were shown to carry the MYSM1 variant in a heterozygous state, indicating a biallelic localization of the variant in the index patient. The variant has not been described before, neither in gnomAD (The Genome Aggregation Database; https://gnomad.broadinstitute.org/) (53) nor in the in-house database of the Institute of Human Genetics with more than 15,000 individuals. Additionally, Sanger sequencing in the asymptomatic siblings showed familial segregation of the MYSM1 variant, consistent with autosomal-recessive inheritance.

To explore the mechanisms for hematopoietic dysfunction, further flow cytometry analyses were performed on patient blood. This demonstrated reduction in protein synthesis rate in many leukocyte populations, as measured using the OPP-incorporation method (Figure 8E and Supplemental Table 8). Furthermore, increase in p53 protein levels was also observed (Figure 8F and Supplemental Table 8), suggesting p53 stress response activation. Together with our other data, these findings provide further support for the common mechanisms of hematopoietic dysfunction in MYSM1 deficiency and ribosomopathy syndromes.

\section{Discussion}

To our knowledge, this work provides the first unbiased genome-wide analysis of MYSM1-regulated genes and demonstrates an essential role for MYSM1 in the induction of $R P$ genes in HSCs. We observe that MYSM1-regulated $R P$ genes are strongly expressed in WT HSCs as compared with downstream MPPs, despite the quiescent state and low protein synthesis rate of steady-state WTs HSCs $(1,2)$. Mysm1 KO results in the loss of this transcriptional signature, with $M y s m 1^{-/-}$HSCs adopting $R P$ gene expression profiles more representative of WT MPP cells. This coincides with HSC dysfunction and loss of hematopoietic homeostasis in $\mathrm{Mysm}^{1^{-1}}$ mice. These findings provide important insights into the functions of MYSM1 as an essential HSC transcriptional regulator, and the specialized mechanisms regulating $R P$ gene expression in HSCs.

These findings represent an important advance in our understanding of the mechanisms underlying HSC dysfunction and hematologic pathologies in Mysm1 deficiency. We and others have shown that, in mice, these phenotypes are mediated by the aberrant activation of p53 in the affected cells $(21,28,29)$; however, the precise mechanisms driving p53 activation remained unclear. Here, we propose that reduced expression of $R P$ genes in Mysm1 deficiency and the resulting ribosomal stress are the major triggers of p53 activation. Ribosomal stress is indeed a well-characterized trigger for p53 activation (10, 50), and studies of ribosomopathy disorders report p53 activation in patients (11-13) and mouse models (47-49). Our profile of the transcriptional state of $M y s m 1^{-1-}$ mouse HSCs provides a model of HSC transcriptome under the conditions of ribosomal stress. It shows that the induction of p53 stress response genes increases progressively with HSC differentiation, coupled with increased cell apoptosis (21). This is consistent with studies of p53 activation in HSCs in response to other forms of stress. Studies of irradiation responses similarly showed reduced expression of proapoptotic p53 target genes in HSCs relative to MPPs and a higher HSC resistance to apoptosis (54-56). The mechanisms underlying these specifics of p53 regulation and cell fate decisions in HSCs in response to genotoxic, ribosomal, and other cellular stresses merit further investigation. Overall, our work draws the mechanistic link between MYSM1 functions as an activator of gene expression in HSCs (14) and the p53-driven hematopoietic failure in Mysm1 deficiency $(21,28,29)$.

Multiple factors suggest conservation of MYSM1 function and the pathogenic mechanisms of MYSM1 deficiency between mice and humans, including high MYSM1 protein homology (87\%), conservation of the core pathways of ribosome biogenesis and p53 stress response, and the similarities in the phenotype of Mysm1-deficient mice and human patients (16-18). In the current work, we characterize a MYSM1 deficiency syndrome patient and report a reduction in protein synthesis rates and increase in p53 protein levels in the patient's blood cells. This, combined with our extensive data from mouse models, indicates that common mechanisms drive disease pathology in MYSM1 deficiency and ribosomopathy syndromes. Interestingly, a homozygous mutation in MYSM1 was recently identified in an exome sequencing study in 1 patient misdiagnosed with DBA (57). Because a significant number of DBA patients do not carry mutations or deletions in established DBA-causing genes $(3,8)$, this together with our current work provides a rationale for screening such patients, as well as patients with other undiagnosed congenital BM failures, for mutations in MYSM1.

Our conclusions may also have implications for other disorders of hematopoiesis, including hematologic malignancies. Inhibitors of ribosome biogenesis and translation are being widely evaluated as novel cancer chemotherapy drugs $(58,59)$, and our work suggests that MYSM1-inhibition may sensitize hematopoietic cells to such agents. Future work will need to address whether targeting MYSM1 can also sensitize 
leukemia stem cells to such inhibitors and will need to evaluate the efficacy of potential combination therapies in hematologic malignancies.

In previous studies, MYSM1 transcriptional functions have been linked to its DUB catalytic activity and protein-to-protein interactions with transcription factors and epigenetic regulators. MYSM1 is one of many nuclear DUBs that can deubiquitinate histone H2AK119ub $(14,60)$, a transcriptionally repressive histone mark deposited on chromatin by the polycomb repressive complex 1 (PRC1) for long-term gene repression during development (42-44). MYSM1 catalytic activity on histone H2AK119ub has been linked to its role as a transcriptional activator of androgen receptor target genes in prostate cancer cell lines (14) and lineage-specification genes including Gfi1, Ebf1, Id2, and Flt3 in hematopoietic cells (20, 22, 24, 27). Our data from hematopoietic progenitors demonstrate that $\mathrm{H} 2 \mathrm{AK} 119 \mathrm{ub}$ levels on $R P$ gene promoters are very low, consistent with the high expression of these genes. With Mysm1 knockdown, RP gene promoters show a reduction in levels of histone H3K27ac, a marker of active transcription, while levels of H2AK119ub do not increase significantly. This suggests that MYSM1 regulates these genes through alternative H2AK119ub-independent mechanisms. Interestingly, MYSM1 has been shown to interact with histone acetylase pCAF, suggesting a possible mechanism through which MYSM1 may modulate H3K27ac levels (14). Other nuclear DUBs with catalytic activity for histone $\mathrm{H} 2 \mathrm{~A}$ have been shown to regulate gene expression and hematopoiesis through diverse mechanisms (60), including catalytic activity on histone H2AK119ub (61), on other ubiquitinated histones (62) and on transcriptional regulators (63), indicating that the molecular mechanisms for MYSM1 regulation of gene expression are likely complex, context-specific, and involve multifaceted interactions.

Recent work characterized aspects of the molecular mechanisms that link $R P$ gene mutations to tissue pathology in ribosomopathies. The studies report that $R P$ mutations may impact cellular physiology by reducing ribosome numbers $(7,64)$ or affecting ribosome composition $(65,66)$. Recent studies also attribute disease pathology to alterations in the relative translation rates of different transcripts (7), linking the reduction in Gatal translation, in particular, to erythroid pathology $(64,67)$. Impact of $R P$ gene mutations on the extraribosomal functions of certain RPs is also considered (68-71). At this time, we cannot rule out that reduced $R P$ expression and protein synthesis in Mysm1-deficient HSCs may affect the differential rates of translation of specific transcripts or impact certain extraribosomal RP functions. However, we observe a full rescue of hematopoietic phenotypes in $M y s m 1^{1^{--}} p 53^{-1-}$ double-KO mice $(28,29)$, despite persistence of $R P$ gene dysregulation. This indicates that the disease pathology in Mysm1 deficiency is driven primarily via 553 activation in response to $R P$ gene dysregulation and ribosomal stress, rather than by other potential molecular and functional effects of $R P$ gene downregulation.

In summary, we demonstrate an essential role for the chromatin binding factor MYSM1 in the normal expression of $R P$ genes and other genes encoding regulators of translation in HSCs. Our work provides insights into the functions of MYSM1 and the specialized mechanisms regulating $R P$ genes in HSCs. Moreover, our work establishes that common mechanisms underlie the hematopoietic dysfunction in human MYSM1 deficiency and ribosomopathy syndromes.

\section{Methods}

Mouse Lines. The Mysm 1 $1^{\text {tmla(KOMP)WTSI }}$ mouse line with over 100-fold reduction in Mysm1 levels was previously described $(19,72)$ and is referred to as $M y s m 1^{-1-}$. The p53-KO and PUMA/Bbc3-KO lines were obtained from The Jackson Laboratory (JAX002101 and JAX011067, respectively). The mice were maintained under specific pathogen-free conditions. All lines were on the C57BL/6 genetic background. Mice used in experiments were 8-14 weeks in age; both males and females were used and sex matched across experimental groups.

Inducible KO mouse models. The Mysm $1^{f l f l}$ mouse line was generated by crossing the Mysm $1^{\mathrm{tmla(KOMP)WTSI}}$ mice to the mouse line with a ubiquitous expression of Flp recombinase Gt(ROSA)26Sor ${ }^{\text {tm1(FLP1)Dym/Wtsi }}$ (73), as described previously (33). This line was subsequently crossed to the Gt(ROSA)26Sor ${ }^{\text {tm1(cre/ERT2) }}$ line to generate Mysm $1^{f l f l}$ CreERT2 mice for tamoxifen-induced deletion of Mysm1 (33). The mice were injected i.p. with tamoxifen (MilliporeSigma, T5648) in sterilized corn oil at $0.15 \mathrm{mg} / \mathrm{gram}$ per injection, with 8 doses in total administered over 16 days. Successful deletion of Mysm1 exon 3 was validated by genotyping of the genomic DNA from hematopoietic and lymphoid organs, and the loss of Mysm1 transcript was further confirmed by qPCR with LSK HSC/MPP cells from mouse BM, as described previously $(21,33)$. $M y s m 1^{\mathrm{fl} /+}$ CreERT2 mice undergoing identical tamoxifen treatment, as well as Mysm $1^{f l}$ mice treated with corn-oil only, were used as controls. 
Tissue culture. Murine pro-B lymphocyte cell line Ba/F3 (DSMZ, ACC 300) was maintained at $0.5 \times$ $10^{6}$ to $2 \times 10^{6}$ cells $/ \mathrm{mL}$ in RPMI-1640 (Wisent) with 10\% FCS (Wisent), $2 \mathrm{mM}$ L-glutamine, $100 \mu \mathrm{g} / \mathrm{mL}$ streptomycin, $100 \mathrm{U} / \mathrm{mL}$ penicillin (Wisent), and 5\% WEHI conditioned media as the source of IL-3. Multipotent hematopoietic progenitor cells HPC7, derived from murine ES-cells via constitutive expression of LIM-homeobox gene LH2, were provided by Leif Carlsson (Umea Center for Molecular Medicine, Umea, Sweden) (38) and previously extensively characterized $(39,40)$. HPC7 cells were cultured at $0.5 \times 10^{6}$ to $2 \times$ $10^{6}$ cells/mL in IMDM (Invitrogen), with 10\% FCS (Thermo Fisher Scientific), $100 \mu \mathrm{g} / \mathrm{mL}$ streptomycin, $100 \mathrm{U} / \mathrm{mL}$ penicillin (Wisent), $7.48 \times 10^{-5} \mathrm{M}$ MTG (M6145, MilliporeSigma), and $100 \mathrm{ng} / \mathrm{mL}$ murine SCF (Shenandoah Biotechnology, Cedarlane).

Three Mysm1 shRNA knockdown (shMysm1) and control shFF Ba/F3 cell lines were previously described (21) and derived through retroviral transduction of the cells with pMSCV-Puro-IRES-GFP shRNA vectors (Addgene) (74). Ba/F3 cell lines stably expressing triple-FLAG-tagged murine MYSM1 were also previously described (21), and HPC7 cells expressing the same construct were derived with similar protocols. All lines were maintained under $2 \mu \mathrm{g} / \mathrm{mL}$ puromycin selection (Wisent). In specific experiments, the cells were treated with inhibitors actinomycin D (SBR00013, MilliporeSigma) and cycloheximide (C7698, MilliporeSigma).

Mouse hematology. Hematology analysis of mouse blood was performed by the Diagnostics Laboratory of the McGill Comparative Medicine Animal Resources Centre (CMARC), as previously described (33).

Cell sorting. Cell sorting protocols were as previously described (21). Briefly, BM was flushed in PBS supplemented with $0.1 \% \mathrm{BSA}$ and $2 \mathrm{mM}$ EDTA, filtered through $40-\mu \mathrm{m}$ cell strainers, and subjected to $\mathrm{RBC}$ lysis in ACK buffer (0.15 M NH biotin anti-mouse Lineage Panel (BioLegend), PE-Cy7 cKit (2B8, BioLegend), APC-Cy7 Sca-1 (D7, BioLegend), FITC CD48 (HM48-1, eBioscience), APC CD150 (TC15-12F12.2, BioLegend), PE Flt3 (A2F10, eBioscience), Brilliant Violet 421 CD34 (RAM34, BD Biosciences), and streptavidin-PECy5 (BioLegend). Cell sorting was performed on FACSAria and analyzed with FACS Diva software (BD Biosciences).

Protein synthesis rate measurements. Analysis of protein synthesis rates was performed using the OPP incorporation method, using previously described protocols $(1,45)$. Briefly, the mice were injected i.p. with OPP (Medchem Source) at $50 \mathrm{mg} / \mathrm{kg}$ in PBS at 1 hour before BM collection. The cells were stained with Fixable Viability Dye eFluor506 (eBioscience) and for appropriate cell surface markers; they were then fixed and permeabilized with BD Cytofix/Cytoperm kit (BD Biosciences) according to the manufacturer's protocols and washed with PBS. This was followed by staining for OPP incorporation using the Click-iT Plus OPP Alexa Fluor 488 Protein Synthesis Assay Kit (Invitrogen) according to the manufacturer's protocols. Samples were analyzed by flow cytometry on FACS Canto II with FACS Diva software (BD Biosciences).

$R N A$ isolation and $q P C R$. RNA isolation from cell lines was carried out with the EZ-10 DNAaway RNA mini-prep kit (BioBasic) according to the manufacturer's protocol. The RNA was quantified with NanoDrop spectrophotometry (Thermo Fisher Scientific) and reverse transcribed with the M-MLV reverse-transcription kit (Invitrogen). RNA isolation from FACS-sorted hematopoietic cells was done using the MagMAX total RNA kit (Ambion) according to the manufacturer's protocol. RNA quality was assessed on Bioanalyzer RNA Pico chips (Agilent), and cDNA was prepared using the qScript XLT cDNA Supermix (Quanta Biosciences) with 2-5 ng RNA inputs. All qPCRs were performed on a StepOnePlus instrument with Power SYBR Mastermix (Applied Biosystems). The primers were purchased from IDT Technologies, and the sequences are provided in Supplemental Table 9.

RNA-Seq. The protocols were as previously described (21). Briefly, RNA was isolated using the MagMAX total RNA kit (Ambion) and quality assessed using Bioanalyzer RNA Pico chips (Agilent). rRNA depletion and library preparation were performed using the SMARTer Stranded RNA-Seq kit (Takara Clontech). The libraries were sequenced on an Illumina HiSeq 2500 sequencer in paired-end 50-bp configuration. The high quality of sequence reads was confirmed using FastQC tool (Babraham Bioinformatics), and low-quality bases were trimmed from read extremities using Trimmomatic v.0.33 (75). Reads were then mapped to the mouse UCSC $\mathrm{mm} 9$ reference assembly using TopHat v2.0.9 in conjunction with Bowtie 1.0.0 algorithms (76-78). Gene expression was quantified by counting the number of uniquely mapped reads with featureCounts using default parameters (79). We retained genes that had an expression level of minimum 5 counts per million (CPM) reads in at least 3 of the samples and performed quantile normalization with the preprocessCore (Bioconductor) package to remove batch effects (80). TMM normalization and differential gene expression analyses were conducted using the edgeR Bioconductor package (81); pairwise comparisons 
were performed between samples across different mouse genotypes. Genes with changes in expression $\geq$ $|1.5|$ fold and Benjamini-Hochberg adjusted $P \leq 0.01$ were considered significant. For data visualization in Integrative Genomics Viewer (IGV) (82), replicates with the same genotype were combined, and bigwig files were generated using a succession of genomeCoverageBed and wigToBigWig tools and scaled per 10 million reads mapping onto exons. GO and disease ontology enrichment analyses on differentially expressed gene clusters were performed with DAVID Bioinformatics Resources 6.8 (83), and GSEA was performed using MSigDB database v5.2 (https://www.gsea-msigdb.org/gsea/msigdb/index.jsp) (34).

ChIP. ChIP was performed as described previously (84), with minor modifications. Briefly, cells were fixed with $1 \%$ formaldehyde in the culture media for 10 minutes at room temperature, followed by addition of 0.125 $\mathrm{M}$ of glycine to stop fixation. Nuclei were extracted with 5 minutes lysis in $0.25 \%$ Triton buffer, followed by 30 minutes in $200 \mathrm{mM} \mathrm{NaCl}$ buffer. Nuclei were resuspended in sonication buffer and sonicated for 12 cycles of 30 seconds with a digital sonifier (Branson Ultrasonics) at $80 \%$, with 30 seconds rest in cooled circulating water.

Beads were prepared overnight with $40 \mu \mathrm{L}$ of Dynabeads Protein G (Invitrogen) conjugated to 3-5 $\mu \mathrm{g}$ of antibodies: anti-Flag M2 (MilliporeSigma, F1804, for MYSM1-FLAG ChIP), anti-H3K27Ac (Abcam, ab4729) or anti-H2AK119Ub (Cell Signaling Technology, D27C4; Supplemental Table 10). Immunoprecipitation was performed by overnight incubation of antibody-bead matrices with sheared chromatin from the equivalent of $5 \times 10^{6}$ cells. For MYSM1-FLAG ChIP, 6 washes were performed with low stringency buffers, while 4 medium-stringency washes were used for histone ChIP. Samples were decross-linked by overnight incubation at $65^{\circ} \mathrm{C}$ in $1 \%$ SDS buffer; following RNaseA and Proteinase K enzymatic treatments, ChIP DNA was purified using Qiaquick PCR Cleanup kit (QIAGEN). ChIP enrichment was quantified using qPCR analysis, with primer sequences provided in Supplemental Table 11. All Ct values were normalized to those of the proopiomelanocortin (Pomc) gene, which serves as a negative binding region. Enrichment was calculated relative to input DNA for MYSM1-FLAG, and relative to total histone $\mathrm{H} 3$ for histone marks.

ChIP-seq libraries were prepared using the Illumina TruSeq kit and sequenced on an Illumina HiSeq 2500 sequencer, with input DNA from the same cells sequenced as negative control. The reads were mapped to the UCSC mouse mm9 reference genome with Bowtie 1.0.0 (85), and chromatin binding sites were identified using peak detection algorithm MACS1.4.1 (86), with comparisons for read enrichment against control input DNA from the same cells. Normalized sequence read density profiles (bigwig) were generated with Homer tool (87) and visualized with IGV (82). GO and disease ontology enrichment analyses on genes associated with MYSM1 ChIP-Seq binding clusters were performed on GREAT 3.0.0 (41) with Basal plus extension option, searching for genes within $2 \mathrm{~kb}$ upstream, $2 \mathrm{~kb}$ downstream, and $200 \mathrm{~kb}$ in distal.

ChIP-Seq and RNA-Seq data consolidation. Full gene annotations were obtained from UCSC mouse $\mathrm{mm} 9$ reference genome. An in-house Python script was developed to load the genomic locations of ChIP-Seq binding sites and RNA-Seq dysregulated genes, and search for gene TSS within a specific distance to each ChIP-Seq binding site.

Exome sequencing, sanger sequencing, and other analyses of patient samples. Blood samples from the patient and his parents were collected, and DNA was extracted using the Gentra Puregene Blood Kit (QIAGEN). Exome sequencing was performed using a Sure Select Human All Exon 60 Mb V6 Kit (Agilent) and a HiSeq4000 (Illumina) as previously described (88). Reads were aligned to the UCSC human reference assembly (hg19) with BWA v.0.5.8. More than $98 \%$ of the exome was covered at least $20 \times$. Single-nucleotide variants and small insertions and deletions were detected with SAMtools v.0.1.7. Copy number variations were called using the software ExomeDepth and Pindel. Variant prioritization was performed based on an autosomal recessive pattern of inheritance (homozygous or compound heterozygous with a minor allele frequency $<0.1 \%$ ), as well as an autosomal dominant pattern of inheritance (heterozygous with a minor allele frequency $<0.001 \%$ that could not be identified in the parents). Sanger sequencing was performed on the index patient and the patient's parents to confirm the described variant, and also on the patient's siblings to show the familial segregation of the variant. Flow cytometry analyses of patient samples are described in detail in Supplemental Methods, with antibodies and gating strategies summarized in Supplemental Tables 12 and 13 and Supplemental Figures 9 and 10.

Data availability. ChIP-Seq and RNA-Seq data are available in the National Center for Biotechnology Information GEO database under the following accession number: GSE150667.

Statistics. Statistical analyses used Prism 7 (GraphPad Inc.), with Student's 2-tailed $t$ test for 2 data sets and 1- or 2-way ANOVA for multiple comparisons; $P<0.05$ was considered significant. Further information on the statistical analyses is provided above for RNA-Seq and ChIP-Seq data, and in the figure legends for other data sets. 
Study approval. All mouse experiments were in accordance with the guidelines of the Canadian Council on Animal Care and protocol AUP-2011-6029, approved by the McGill Animal Care Committee.

The patient was referred to the Children's Hospital Schwabing of the Technische Universitaet Muenchen (TUM) and the Munich Municipal Hospital Group (MMHG), as well as to the Institute of Human Genetics at the TUM (Munich, Germany). Genetic analyses of the samples was approved by the local Ethics Committee of the TUM and performed according to the standard of the Declaration of Helsin$\mathrm{ki}$, after written informed consent from both parents. Other experiments were approved by IRB Assurance no. IRB00002927, CHU Sainte-Justine IRB no. 1, Montreal, Canada; protocol no. 4181; parent institution IORG0002378 - CHU Sainte-Justine; assurance no. FWA00021692.

\section{Author contributions}

The experiments were performed by JIB, AF, JCP, HW, YHL, CHF, JT, and AN. The data were analyzed and prepared for publication by HW, DL, AN, JIB, and AF. The manuscript was written by AN, DL, HW, JIB, and AF. The experiments were designed by DL, AN, and JIB. The work was supervised by DL, AN, and PG. GSL, TB, UB, PMC, and TTMN provided the patient blood sample, clinical and/or genetic data, and contributed to manuscript preparation. Two first authors JIB and HW are listed based on the alphabetical order of their last names.

\section{Acknowledgments}

This work was funded by the Canadian Institutes of Health Research (CIHR, project grant PJT153016, operating grant MOP-123403) and supported by the Canadian Foundation for Innovation (CFI). AN is a Canada Research Chair Tier II in Hematopoiesis and Lymphocyte Differentiation. HCW is a recipient of the Fonds de Recherche du Québec Santé (FRQS) Masters Training Studentship. AF is a recipient of the Frederick Banting Tri-Council Scholarship and was previously supported by FRQS Masters Training Studentship and the Max \& Jane Childress Entrance Fellowship from the Department of Physiology of McGill University. JCP was a recipient of an Internal Studentship from the Faculty of Medicine of McGill University. YHL is a recipient of the Cole Foundation Studentship and was previously supported by the Frederick Banting Tri-Council Graduate Scholarship. DL was supported by a postdoctoral fellowship from the CIHR Neuroinflammation Training Program and from the FRQS. PMC is a recipient of Clinician-Scientist awards from the CIHR and the FRQS. UB is a recipient of research grants from the Helmholtz Center Munich (HMGU), the German Center for Infection Research (DZIF), the Lost Voices Foundation, and the Weidenhammer-Zoebele Foundation. The collaboration was facilitated by GeneMatcher (89).

We thank Odile Neyret and Myriam Rondeau from the Molecular Biology and Functional Genomics Facility of the Institut de Recherches Cliniques de Montréal (IRCM) for next-generation sequencing. Bioinformatics data analyses used the resources of Compute Canada and Calcul Québec. The flow cytometry and cell sorting were performed at the Cell Vision Core Facility of the McGill Life Science Complex, with the support of the Canadian Foundation for Innovation, or at the IRCM Flow Cytometry Core Facility. We thank Julien Leconte, Eric Massicotte, and Julie Lord for their assistance with cell sorting. Hematology analyses of mouse blood were performed at the Diagnostics Laboratory of the CMARC. We thank Marie-Josee Benoit, Catherine Gagné, Tanya Koch, and other CMARC staff for mouse colony management, as well as Hieu Nguyen, Sarah Elliott, Yurim Park, and Shiyang Shen for mouse genotyping. HPC7 cells were provided by Leif Carlsson (Umea Center for Molecular Medicine, Sweden). We thank Daniela Schenk (Munich Municipal Hospital Group, Munich, Germany) for patient hematology data and helpful discussions.

Address correspondence to: Anastasia Nijnik, 368 Bellini Life Sciences Complex, 3649 Promenade Sir William Osler, McGill University, H3G 0B1 Montreal, Quebec, Canada. Phone: 514.398.5567; Email: anastasia.nijnik@mcgill.ca. Or to: David Langlais, Room 4203, 740 Avenue Dr Penfield, McGill University Genome Centre, Montreal, Quebec, Canada, H3A 0G1. Phone: 514.398.5844; Email: david. langlais@mcgill.ca. 
1. Signer RA, Magee JA, Salic A, Morrison SJ. Haematopoietic stem cells require a highly regulated protein synthesis rate. Nature. 2014;509(7498):49-54.

2. Barna M, Ruggero D. Tailor made protein synthesis for HSCs. Cell Stem Cell. 2014;14(4):423-424.

3. Da Costa L, Narla A, Mohandas N. An update on the pathogenesis and diagnosis of Diamond-Blackfan anemia. F1000Res. 2018;7:F1000 Faculty Rev-1350.

4. Huang JN, Shimamura A. Clinical spectrum and molecular pathophysiology of Shwachman-Diamond syndrome. Curr Opin Hematol. 2011;18(1):30-35.

5. Barlow JL, Drynan LF, Trim NL, Erber WN, Warren AJ, McKenzie AN. New insights into 5q- syndrome as a ribosomopathy. Cell Cycle. 2010;9(21):4286-4293.

6. Ebert BL, et al. Identification of RPS14 as a 5q- syndrome gene by RNA interference screen. Nature. 2008;451(7176):335-339.

7. Mills EW, Green R. Ribosomopathies: There's strength in numbers. Science. 2017;358(6363):eaan2755.

8. Narla A, Ebert BL. Ribosomopathies: human disorders of ribosome dysfunction. Blood. 2010;115(16):3196-3205.

9. Ito E, Konno Y, Toki T, Terui K. Molecular pathogenesis in Diamond-Blackfan anemia. Int J Hematol. 2010;92(3):413-418.

10. Golomb L, Volarevic S, Oren M. p53 and ribosome biogenesis stress: the essentials. FEBS Lett. 2014;588(16):2571-2579.

11. Dutt $\mathrm{S}$, et al. Haploinsufficiency for ribosomal protein genes causes selective activation of p53 in human erythroid progenitor cells. Blood. 2011;117(9):2567-2576.

12. Caceres G, et al. TP53 suppression promotes erythropoiesis in del(5q) MDS, suggesting a targeted therapeutic strategy in lenalidomide-resistant patients. Proc Natl Acad Sci USA. 2013;110(40):16127-16132.

13. Pellagatti A, et al. Induction of $\mathrm{p} 53$ and up-regulation of the $\mathrm{p} 53$ pathway in the human $5 \mathrm{q}$ - syndrome. Blood. 2010;115(13):2721-2723.

14. Zhu P, et al. A histone H2A deubiquitinase complex coordinating histone acetylation and H1 dissociation in transcriptional regulation. Mol Cell. 2007;27(4):609-621.

15. Fiore A, et al. Deubiquitinase MYSM1 in the Hematopoietic System and beyond: A Current Review. Int J Mol Sci. 2020;21(8):E3007.

16. Alsultan A, Shamseldin HE, Osman ME, Aljabri M, Alkuraya FS. MYSM1 is mutated in a family with transient transfusion-dependent anemia, mild thrombocytopenia, and low NK- and B-cell counts. Blood. 2013;122(23):3844-3845.

17. Le Guen T, et al. An in vivo genetic reversion highlights the crucial role of Myb-Like, SWIRM, and MPN domains 1 (MYSM1) in human hematopoiesis and lymphocyte differentiation. J Allergy Clin Immunol. 2015;136(6):1619-1626.e5.

18. Bahrami E, et al. Myb-like, SWIRM, and MPN domains 1 (MYSM1) deficiency: Genotoxic stress-associated bone marrow failure and developmental aberrations. J Allergy Clin Immunol. 2017;140(4):1112-1119.

19. Nijnik A, et al. The critical role of histone H2A-deubiquitinase Mysm1 in hematopoiesis and lymphocyte differentiation. Blood. 2012;119(6):1370-1379.

20. Wang T, et al. The control of hematopoietic stem cell maintenance, self-renewal, and differentiation by Mysm1-mediated epigenetic regulation. Blood. 2013;122(16):2812-2822.

21. Belle JI, et al. Repression of p53-target gene Bbc3/PUMA by MYSM1 is essential for the survival of hematopoietic multipotent progenitors and contributes to stem cell maintenance. Cell Death Differ. 2016;23(5):759-775.

22. Jiang XX, et al. Control of B cell development by the histone H2A deubiquitinase MYSM1. Immunity. 2011;35(6):883-896.

23. Huang XF, et al. Mysm1 is required for interferon regulatory factor expression in maintaining HSC quiescence and thymocyte development. Cell Death Dis. 2016;7(6):e2260.

24. Nandakumar V, Chou Y, Zang L, Huang XF, Chen SY. Epigenetic control of natural killer cell maturation by histone H2A deubiquitinase, MYSM1. Proc Natl Acad Sci USA. 2013;110(41):E3927-E3936.

25. Förster M, et al. MYSM1-dependent checkpoints in B cell lineage differentiation and B cell-mediated immune response. J Leukoc Biol. 2017;101(3):643-654.

26. Förster M, et al. A role for the histone H2A deubiquitinase MYSM1 in maintenance of CD8 $8^{+} \mathrm{T}$ cells. Immunology. 2017;151(1):110-121.

27. Won $\mathrm{H}$, et al. Epigenetic control of dendritic cell development and fate determination of common myeloid progenitor by Mysm1. Blood. 2014;124(17):2647-2656.

28. Belle JI, et al. p53 mediates loss of hematopoietic stem cell function and lymphopenia in Mysm1 deficiency. Blood. 2015;125(15):2344-2348.

29. Gatzka M, et al. Interplay of H2A deubiquitinase 2A-DUB/Mysm1 and the p19(ARF)/p53 axis in hematopoiesis, early T-cell development and tissue differentiation. Cell Death Differ. 2015;22(9):1451-1462.

30. Petrov JC, Nijnik A. Mysm1 expression in the bone marrow niche is not essential for hematopoietic maintenance. Exp Hematol. 2017;47:76-82.e3.

31. Wilson A, et al. Hematopoietic stem cells reversibly switch from dormancy to self-renewal during homeostasis and repair. Cell. 2008;135(6):1118-1129.

32. Cabezas-Wallscheid N, et al. Identification of regulatory networks in HSCs and their immediate progeny via integrated proteome, transcriptome, and DNA methylome analysis. Cell Stem Cell. 2014;15(4):507-522.

33. Förster M, Belle JI, Petrov JC, Ryder EJ, Clare S, Nijnik A. Deubiquitinase MYSM1 Is Essential for Normal Fetal Liver Hematopoiesis and for the Maintenance of Hematopoietic Stem Cells in Adult Bone Marrow. Stem Cells Dev. 2015;24(16):1865-1877.

34. Subramanian A, et al. Gene set enrichment analysis: a knowledge-based approach for interpreting genome-wide expression profiles. Proc Natl Acad Sci USA. 2005;102(43):15545-15550.

35. Simsek T, et al. The distinct metabolic profile of hematopoietic stem cells reflects their location in a hypoxic niche. Cell Stem Cell. 2010;7(3):380-390.

36. Suda T, Takubo K, Semenza GL. Metabolic regulation of hematopoietic stem cells in the hypoxic niche. Cell Stem Cell. 2011;9(4):298-310.

37. Hüttmann A, et al. Gene expression profiles in murine hematopoietic stem cells revisited: analysis of cDNA libraries reveals high levels of translational and metabolic activities. Stem Cells. 2006;24(7):1719-1727.

38. Pinto do OP, Kolterud A, Carlsson L. Expression of the LIM-homeobox gene LH2 generates immortalized steel factor-depen- 
dent multipotent hematopoietic precursors. EMBO J. 1998;17(19):5744-5756.

39. Wilson NK, et al. Integrated genome-scale analysis of the transcriptional regulatory landscape in a blood stem/progenitor cell model. Blood. 2016;127(13):e12-e23

40. Wilson NK, et al. Combinatorial transcriptional control in blood stem/progenitor cells: genome-wide analysis of ten major transcriptional regulators. Cell Stem Cell. 2010;7(4):532-544.

41. McLean CY, et al. GREAT improves functional interpretation of cis-regulatory regions. Nat Biotechnol. 2010;28(5):495-501.

42. Vidal M, Starowicz K. Polycomb complexes PRC1 and their function in hematopoiesis. Exp Hematol. 2017;48:12-31.

43. Steffen PA, Ringrose L. What are memories made of? How Polycomb and Trithorax proteins mediate epigenetic memory. Nat Rev Mol Cell Biol. 2014;15(5):340-356.

44. Wang H, et al. Role of histone H2A ubiquitination in Polycomb silencing. Nature. 2004;431(7010):873-878.

45. Liu J, Xu Y, Stoleru D, Salic A. Imaging protein synthesis in cells and tissues with an alkyne analog of puromycin. Proc Natl Acad Sci USA. 2012;109(2):413-418.

46. Narla A, Hurst SN, Ebert BL. Ribosome defects in disorders of erythropoiesis. Int J Hematol. 2011;93(2):144-149.

47. Barlow JL, et al. A p53-dependent mechanism underlies macrocytic anemia in a mouse model of human 5q- syndrome. Nat Med. 2010;16(1):59-66.

48. Jaako P, et al. Disruption of the 5S RNP-Mdm2 interaction significantly improves the erythroid defect in a mouse model for Diamond-Blackfan anemia. Leukemia. 2015;29(11):2221-2229.

49. Jaako P, et al. Mice with ribosomal protein S19 deficiency develop bone marrow failure and symptoms like patients with Diamond-Blackfan anemia. Blood. 2011;118(23):6087-6096.

50. Zhang Y, Lu H. Signaling to p53: ribosomal proteins find their way. Cancer Cell. 2009;16(5):369-377.

51. Nanda A, Al-Abboh H, Zahra A, Al-Sabah H, Gupta A, Adekile AD. Neutrophilic Panniculitis in a child with MYSM1 deficiency. Pediatr Dermatol. 2019;36(2):258-259.

52. Fargo JH, et al. Erythrocyte adenosine deaminase: diagnostic value for Diamond-Blackfan anaemia. Br J Haematol. 2013;160(4):547-554

53. Lek M, et al. Analysis of protein-coding genetic variation in 60,706 humans. Nature. 2016;536(7616):285-291.

54. Mohrin M, et al. Hematopoietic stem cell quiescence promotes error-prone DNA repair and mutagenesis. Cell Stem Cell. 2010;7(2):174-185.

55. Labi V, et al. Apoptosis of leukocytes triggered by acute DNA damage promotes lymphoma formation. Genes Dev. 2010;24(15):1602-1607.

56. Beerman I, Seita J, Inlay MA, Weissman IL, Rossi DJ. Quiescent hematopoietic stem cells accumulate DNA damage during aging that is repaired upon entry into cell cycle. Cell Stem Cell. 2014;15(1):37-50.

57. Ulirsch JC, et al. The Genetic Landscape of Diamond-Blackfan Anemia. Am J Hum Genet. 2018;103(6):930-947.

58. Brighenti E, Treré D, Derenzini M. Targeted cancer therapy with ribosome biogenesis inhibitors: a real possibility? Oncotarget. 2015;6(36):38617-38627.

59. Pelletier J, Thomas G, Volarević S. Ribosome biogenesis in cancer: new players and therapeutic avenues. Nat Rev Cancer. 2018;18(1):51-63.

60. Belle JI, Nijnik A. H2A-DUBbing the mammalian epigenome: expanding frontiers for histone H2A deubiquitinating enzymes in cell biology and physiology. Int J Biochem Cell Biol. 2014;50:161-174.

61. Gu Y, et al. The histone H2A deubiquitinase Usp16 regulates hematopoiesis and hematopoietic stem cell function. Proc Natl Acad Sci USA. 2016;113(1):E51-E60.

62. Lancini C, et al. Tight regulation of ubiquitin-mediated DNA damage response by USP3 preserves the functional integrity of hematopoietic stem cells. J Exp Med. 2014;211(9):1759-1777.

63. Dey A, et al. Loss of the tumor suppressor BAP1 causes myeloid transformation. Science. 2012;337(6101):1541-1546.

64. Khajuria RK, et al. Ribosome Levels Selectively Regulate Translation and Lineage Commitment in Human Hematopoiesis. Cell. 2018;173(1):90-103.e19.

65. Shi Z, Barna M. Translating the genome in time and space: specialized ribosomes, RNA regulons, and RNA-binding proteins. Annu Rev Cell Dev Biol. 2015;31:31-54.

66. Shi Z, et al. Heterogeneous Ribosomes Preferentially Translate Distinct Subpools of mRNAs Genome-wide. Mol Cell. 2017;67(1):71-83.e7.

67. Ludwig LS, et al. Altered translation of GATA1 in Diamond-Blackfan anemia. Nat Med. 2014;20(7):748-753.

68. O'Leary MN, et al. The ribosomal protein Rp122 controls ribosome composition by directly repressing expression of its own paralog, Rp12211. PLoS Genet. 2013;9(8):e1003708.

69. Xue S, Tian S, Fujii K, Kladwang W, Das R, Barna M. RNA regulons in Hox 5' UTRs confer ribosome specificity to gene regulation. Nature. 2015;517(7532):33-38.

70. Solanki NR, et al. Rp122 Loss Selectively Impairs $\alpha \beta$ T Cell Development by Dysregulating Endoplasmic Reticulum Stress Signaling. J Immunol. 2016;197(6):2280-2289.

71. Fahl SP, Harris B, Coffey F, Wiest DL. Rp122 Loss Impairs the Development of B Lymphocytes by Activating a p53-Dependent Checkpoint. J Immunol. 2015;194(1):200-209.

72. Okaty BW, et al. Multi-Scale Molecular Deconstruction of the Serotonin Neuron System. Neuron. 2015;88(4):774-791.

73. Farley FW, Soriano P, Steffen LS, Dymecki SM. Widespread recombinase expression using FLPeR (flipper) mice. Genesis. 2000;28(3-4):106-110.

74. Mavrakis KJ, et al. Genome-wide RNA-mediated interference screen identifies miR-19 targets in Notch-induced T-cell acute lymphoblastic leukaemia. Nat Cell Biol. 2010;12(4):372-379.

75. Bolger AM, Lohse M, Usadel B. Trimmomatic: a flexible trimmer for Illumina sequence data. Bioinformatics. 2014;30(15):2114-2120.

76. Kim D, Pertea G, Trapnell C, Pimentel H, Kelley R, Salzberg SL. TopHat2: accurate alignment of transcriptomes in the presence of insertions, deletions and gene fusions. Genome Biol. 2013;14(4):R36.

77. Langmead B, Trapnell C, Pop M, Salzberg SL. Ultrafast and memory-efficient alignment of short DNA sequences to the human genome. Genome Biol. 2009;10(3):R25. 
78. Trapnell C, Pachter L, Salzberg SL. TopHat: discovering splice junctions with RNA-Seq. Bioinformatics. 2009;25(9):1105-1111

79. Liao Y, Smyth GK, Shi W. featureCounts: an efficient general purpose program for assigning sequence reads to genomic features. Bioinformatics. 2014;30(7):923-930

80. Bolstad B. preprocessCore: A collection of pre-processing functions. GitHub. https://github.com/bmbolstad/preprocessCore Accessed June 3, 2020.

81. Robinson MD, Oshlack A. A scaling normalization method for differential expression analysis of RNA-seq data. Genome Biol. 2010;11(3):R25.

82. Thorvaldsdóttir H, Robinson JT, Mesirov JP. Integrative Genomics Viewer (IGV): high-performance genomics data visualization and exploration. Brief Bioinformatics. 2013;14(2):178-192.

83. Huang DW, et al. The DAVID Gene Functional Classification Tool: a novel biological module-centric algorithm to functionally analyze large gene lists. Genome Biol. 2007;8(9):R183.

84. Langlais D, Couture C, Balsalobre A, Drouin J. The Stat3/GR interaction code: predictive value of direct/indirect DNA recruitment for transcription outcome. Mol Cell. 2012;47(1):38-49.

85. Langmead B, Trapnell C, Pop M, Salzberg SL. Ultrafast and memory-efficient alignment of short DNA sequences to the human genome. Genome Biol. 2009;10(3):R25.

86. Zhang Y, et al. Model-based analysis of ChIP-Seq (MACS). Genome Biol. 2008;9(9):R137.

87. Heinz S, et al. Simple combinations of lineage-determining transcription factors prime cis-regulatory elements required for macrophage and B cell identities. Mol Cell. 2010;38(4):576-589.

88. Kremer LS, et al. Genetic diagnosis of Mendelian disorders via RNA sequencing. Nat Commun. 2017;8:15824

89. Sobreira N, Schiettecatte F, Valle D, Hamosh A. GeneMatcher: a matching tool for connecting investigators with an interest in the same gene. Hum Mutat. 2015;36(10):928-930. 